\title{
Classification of Plant Leaves Using New Compact Convolutional Neural Network Models
}

\author{
Shivali Amit Wagle ${ }^{1}\left(\mathbb{D}\right.$, R. Harikrishnan ${ }^{1, *(\mathbb{D}}$, Sawal Hamid Md Ali ${ }^{2, *(\mathbb{D})}$ and Mohammad Faseehuddin ${ }^{1}$ (D) \\ 1 E\&TC Department, Symbiosis Institute of Technology, Symbiosis International Deemed University, \\ Pune 412115, India; kulkarni_shivali@yahoo.co.in (S.A.W.); faseehuddin03@gmail.com (M.F.) \\ 2 Department of Electrical, Electronic and Systems Engineering, Universiti Kebangsaan Malaysia, \\ Bangi 43600, Malaysia \\ * Correspondence: dr.rhareish@gmail.com (R.H.); sawal@ukm.edu.my (S.H.M.A.)
}

\section{check for} updates

Citation: Wagle, S.A.; Harikrishnan, R.; Ali, S.H.M.; Mohammad, F. Classification of Plant Leaves Using New Compact Convolutional Neural Network Models. Plants 2022, 11, 24. https://doi.org/10.3390/ plants11010024

Academic Editors: Laura-Jayne Gardiner and Ritesh Krishna

Received: 25 October 2021 Accepted: 29 November 2021 Published: 22 December 2021

Publisher's Note: MDPI stays neutral with regard to jurisdictional claims in published maps and institutional affiliations.

Copyright: () 2021 by the authors. Licensee MDPI, Basel, Switzerland. This article is an open access article distributed under the terms and conditions of the Creative Commons Attribution (CC BY) license (https:/ / creativecommons.org/licenses/by/ $4.0 /)$.

\begin{abstract}
Precision crop safety relies on automated systems for detecting and classifying plants. This work proposes the detection and classification of nine species of plants of the PlantVillage dataset using the proposed developed compact convolutional neural networks and AlexNet with transfer learning. The models are trained using plant leaf data with different data augmentations. The data augmentation shows a significant improvement in classification accuracy. The proposed models are also used for the classification of 32 classes of the Flavia dataset. The proposed developed N1 model has a classification accuracy of $99.45 \%, \mathrm{~N} 2$ model has a classification accuracy of $99.65 \%, \mathrm{~N} 3$ model has a classification accuracy of $99.55 \%$, and AlexNet has a classification accuracy of $99.73 \%$ for the PlantVillage dataset. In comparison to AlexNet, the proposed models are compact and need less training time. The proposed N1 model takes $34.58 \%$, the proposed N2 model takes $18.25 \%$, and the N3 model takes $20.23 \%$ less training time than AlexNet. The N1 model and N3 models are size 14.8 MB making it $92.67 \%$ compact, and the N2 model is $29.7 \mathrm{MB}$ which makes it $85.29 \%$ compact as compared to AlexNet. The proposed models are giving good accuracy in classifying plant leaf, as well as diseases in tomato plant leaves.
\end{abstract}

Keywords: classification; compact model; convolutional neural network; plant leaf

\section{Introduction}

Plants provide food for all living things, making them the backbone of the ecosystem [1]. Plant species are valuable as medicine, foodstuff, and also for industrial applications. Some plants are at risk of extinction. So, it is imperative to set up a database for plant protection. Manual examination of the plant with the naked eye is the most basic or conventional technique. This procedure, involves constant supervision of a wide range of farm areas by experts [2,3]. This is a time-consuming and costly procedure. To achieve plant protection, the classification of the plant plays an essential role [4]. Plants leaves are easily accessible and prominent parts, unlike flowers which are available for a shorter period. The leaves are, therefore, a good choice for automatic plant classification. The leaves are important in exploring the genetic relationship of plants and the explanation of plant development. However, given a large number of species, plant identification, even for botanists, is a relatively difficult task $[5,6]$. The leaf recognition technology was followed by botanists for classifying specific plant species. Plants generally have distinctive features that differ in many aspects, such as texture, shape, color, and size; they are different [7]. In the last couple of years, different "Computer-Aided Detection" (CAD) methods are deployed for leaf based plant recognition owing to their high classification accuracy [8,9].

The interdisciplinary approach of plant classification combines botanical data, and the concept of species with computer solutions [10]. Recent advances in science and technology allow computer vision approach to help botanists to identify plants. Computer vision researchers have used leaves to classify plants as a comparative tool [11]. From the machine 
learning point of view, the classification problem can be addressed by adopting a new quick solution, which will bring experts, farmers, decision-makers, and strategists into a single chorus [12].

In recent years, evolutionary neural networks have attracted much attention of the researchers because of their ability to give superior image classification accuracy. They combine the neural network and computation to solve any problem. Krizhevsky et al. [13] set a record of $10.9 \%$ more classification accuracy compared to the second-best entry in ImageNet in the 2012 Large Scaled Challenge for Visual Recognition. Advances in the processing of images provided various preprocessing techniques for the extraction of images. Feature extraction is the step taken to identify discriminatory characteristics that form the basis for classification. The classification task can be performed with multiple learning technologies such as "Support Vector Machines" (SVM), Naïve Baye, "K-Nearest Neighbor" (KNN), and "Convolutional Neural Network" (CNN) [14].

Deep learning is a subset of machine learning that consists of a set of algorithms for modeling high-level data abstractions using a deep graph with multiple processing layers that include linear and non-linear transformations [15]. CNNs are well-suited for image classification tasks due to their close relationship between layers and spatial information, which explains their popularity in recent plant classifiers.

Several studies have found that image-based assessment methods produce more accurate and consistent results than human visual assessments [16]. A lot of work has been completed to classify things using various techniques. Lecun et al. [17] introduced the basic deep learning tool of CNN as an introduction to deep learning model techniques in the field of classification and detection. In recent years, deep learning models have been used to a small extent in agriculture. CNNs are a form of a dynamic model that aids classification applications. For classification, there are many CNN models, such as AlexNet [13], GoogLeNet [18], ResNet50, ResNet18, ResNet101 [19], VGG16, VGG19 [20], DenseNet [21], SqueezeNet [22], and others.

Mohanty et al. [23] classified 14 different plant leaves using AlexNet and GoogLeNet, with an accuracy of $99.27 \%$ and $99.34 \%$, respectively. The authors used different input data, such as color images, segmented images, and grayscale images separately. Dyrmann et al. [24] classified the plant leaf data with a CNN model and achieved an accuracy of $86.2 \%$. Barré et al. [25] in their work for plant leaf classification, used LeafSnap, Foliage, and Flavia dataset for classification of different classes with their proposed model LeafNet. A total of 184 classes of LeafSnap were classified with an accuracy of $86.3 \%$, and 60 classes of the Foliage dataset were classified with an accuracy of $95.8 \%$. They achieved the performance accuracy of $97.9 \%$ for the Flavia dataset with 32 classes. A deep CNN model [10] with "Multilayer Perceptron" (MLP) classifier achieved 97.7\% accuracy and improved to $98.1 \%$ accuracy with SVM classifier for the MalayaKew dataset with 44 classes. Haque et al. [26] have presented work for plant classification that uses geometric features in preprocessing and achieved an accuracy of $90 \%$ for the classification of 10 plant species of Flavia dataset. Gao et al. [1] achieved an accuracy of $84.2 \%$ in a LifeCLEF Plant Identification Task with their proposed 3SN Siamese network that learns from spatial and structural features for the leaf classification task. The recognition of plant family and further identifying the plant class for the four datasets was performed with two ways attention CNN model by [27].

In the preparation of Ayurvedic medicines, the identification and classification of medicinal plants play an essential role. In addition, it is important for farmers, botanists, practitioners, the forest department's offices, and those involved in the preparation of Ayurvedic medicines for a correct classification of medicinal plants. Medicinal plant classification by [28] with AlexNet model achieved an accuracy of $94.87 \%$, and for the Ayurleaf CNN model, the accuracy is $95.06 \%$. Duong-Trung et al. [12] achieved $98.5 \%$ classification accuracy with the MobileNet model for 20 species of self-collected medicinal plant data. 
Liu et al. [29] proposed a ten-layer CNN model for the classification of plant leaf and achieved an accuracy of $87.92 \%$ for the 32 classes. ResNet model gave the classification accuracy of $93.09 \%$ for plant identification with LeafSnap dataset [9]. Plant leaf classification was completed by [5] on the images captured by Silva et al. [30] using an Apple iPad device. The Deep Neural network (DNN) model shows $91.17 \%$ accuracy, and with the CNN model, the accuracy is improved to $95.58 \%$. The classification of plant leaf with the complex background was completed by [6] on the images captured through mobile phones. The classification accuracy is $91.5 \%, 92.4 \%$, and $89.6 \%$ for VGG16, VGG 19, and the Inception ResNetV2 model, respectively. For the identification of the berry plants, Ref. [14] used the AlexNet model and achieved an accuracy of $97.80 \%$ for the three classes of self-collected data of berry plants. A comparative analysis of the work related to the classification of plants is shown in Table 1.

Table 1. A comparative analysis of the work related to the classification of plants.

\begin{tabular}{|c|c|c|c|c|c|}
\hline Ref. No. & Objective & Dataset & $\begin{array}{l}\text { Number of } \\
\text { Classes }\end{array}$ & Model & Accuracy \\
\hline Dyrmann et al. [24] & Plant leaf classification & Six different datasets & 22 & $\mathrm{CNN}$ & $86.20 \%$ \\
\hline \multirow{2}{*}{ Mohanty et al. [23] } & \multirow{2}{*}{ Identify 14 crop species } & \multirow{2}{*}{ PlantVillage } & 38 & AlexNet & $99.27 \%$ \\
\hline & & & 38 & GoogLeNet & $99.34 \%$ \\
\hline \multirow{3}{*}{ Barré et al. [25] } & \multirow{3}{*}{ plant identification system } & \multirow{3}{*}{ LeafSnap } & 184 & LeafNet & $86.30 \%$ \\
\hline & & & 60 & LeafNet & $95.80 \%$ \\
\hline & & & 32 & LeafNet & $97.90 \%$ \\
\hline \multirow{2}{*}{ Lee et al. [10] } & \multirow{2}{*}{ Plant leaf classification } & \multirow{2}{*}{ Malayakew } & 44 & Deep CNN (D1) MLP & $97.70 \%$ \\
\hline & & & 44 & Deep CNN (D1) SVM (linear) & $98.10 \%$ \\
\hline Gao et al. [1] & Leaf Identification & LifeCLEF 2015 & 30 & $3 \mathrm{SN}$ & $84.20 \%$ \\
\hline \multirow{2}{*}{ Dileep and Pournami [28] } & \multirow{2}{*}{ Medicinal plant classification } & \multirow{2}{*}{ AyurLeaf } & 40 & AlexNet & $94.87 \%$ \\
\hline & & & 40 & Ayurleaf CNN & $95.06 \%$ \\
\hline Duong-Trung et al. [12] & Medicinal plant classification & Own data & 20 & MobileNet & $98.50 \%$ \\
\hline Liu et al. [29] & $\begin{array}{l}\text { Classification of } 32 \text { different } \\
\text { plant leaves }\end{array}$ & Flavia & 32 & Ten-layer CNN model & $87.92 \%$ \\
\hline Bodhwani et al. [9] & Plant Identification & LeafSnap & 180 & ResNet & $93.09 \%$ \\
\hline \multirow{2}{*}{ Tiwari [5] } & \multirow{2}{*}{ Plant leaf classification } & \multirow{2}{*}{ Dataset collected by [30] } & 30 & DNN & $91.17 \%$ \\
\hline & & & 30 & $\mathrm{CNN}$ & $95.58 \%$ \\
\hline \multirow{3}{*}{ Yang et al. [6] } & \multirow{3}{*}{ Classification of plant leaf } & \multirow{3}{*}{ Own data } & 15 & VGG16 & $91.50 \%$ \\
\hline & & & 15 & VGG19 & $92.40 \%$ \\
\hline & & & 15 & Inception- ResNetV2 & $89.60 \%$ \\
\hline Villaruz [14] & Identification of berry plants & Own data & 3 & AlexNet & $97.80 \%$ \\
\hline
\end{tabular}

Once the classification of the plant is completed, further work can be extended to the classification of disease. The VGG16 model was trained with transfer learning for the apple leaf disease and yielded an overall accuracy of $90.4 \%$ [16]. With the augmented dataset of 14828 images of tomato leaves, Ref. [31] achieved an accuracy of $98.66 \%$ for AlexNet and $98.18 \%$ for the VGG16 model. A small CNN was proposed by [32] for the classification of the plant into a healthy or diseased category and achieved an accuracy of $96.6 \%$. The classification accuracy achieved by [33] for tomato plant disease with the laboratory data is 98.50\% for the VGG16 model, $98.30 \%$ for the VGG19 model, $99.40 \%$ for ResNet model, and $99.60 \%$ for the Inception V3 model. The proposed model of [34] outperformed AlexNet and VGG16 with an accuracy of $99.45 \%$ in the classification of tomato plant leaf, with an accuracy of $90.1 \%$.

Guava fruit diseases classification by [35] achieved an accuracy of $99 \%$ for the Bagged Tree classifier on a set of RGB, HSV, and LBP features. Detection of Cassava plant disease by [36] achieved an accuracy of $96.75 \%$ with the deep residual neural network model. Alli et al. [37] used a data augmentation method to achieve an accuracy of $99.7 \%$ for cassava plant disease classification using MobileNetV2. Pearl millet disease classification 
with an automated method of collecting the pearl millet data from the farm and classifying the disease with a Custom-Net model with an accuracy of $98.78 \%$ [38]. A comparative analysis of the work related to plant disease classification is shown in Table 2.

Table 2. A comparative analysis of the work related to the classification of plants disease.

\begin{tabular}{|c|c|c|c|c|}
\hline Ref. No. & Model & Dataset & Objective & Future Scope \\
\hline Wang et al. [16] & VGG16 & PV & Apple black rot disease severity & $\begin{array}{l}\text { More data at various stages of disease } \\
\text { can be used to improve accuracy. }\end{array}$ \\
\hline Mohanty et al. [23] & AlexNet, GoogLeNet & PV & $\begin{array}{l}\text { Identify } 14 \text { crop species and } \\
26 \text { diseases }\end{array}$ & $\begin{array}{l}\text { Image data from a smartphone can be } \\
\text { supplemented with location and time } \\
\text { information to improve accuracy even } \\
\text { further. }\end{array}$ \\
\hline Brahimi et al. [31] & AlexNet, GoogLeNet & PV & $\begin{array}{l}\text { Tomato plant disease classifica- } \\
\text { tion }\end{array}$ & $\begin{array}{l}\text { Reduce the computation and the size of } \\
\text { deep models }\end{array}$ \\
\hline Bharali et al. [32] & CNN model & Google images & $\begin{array}{l}\text { Classifying into healthy and dis- } \\
\text { ease class for different plant }\end{array}$ & $\begin{array}{l}\text { Larger datasets and more complex net- } \\
\text { works can be created to assess perfor- } \\
\text { mance and improve accuracy. }\end{array}$ \\
\hline Ahmad et al. [33] & $\begin{array}{l}\text { VGG16, VGG19, ResNet } \\
\text { Inception-V3 }\end{array}$ & Own data & $\begin{array}{l}\text { Classification of Tomato plant } \\
\text { disease }\end{array}$ & $\begin{array}{l}\text { Optimize these models for better perfor- } \\
\text { mance on real-world field-based data. }\end{array}$ \\
\hline $\begin{array}{l}\text { Anadhakrishnan } \\
\text { et al. [34] }\end{array}$ & $\begin{array}{l}\text { AlexNet, VGG16, LeNet, } \\
\text { ResNet, CNN model }\end{array}$ & PV & $\begin{array}{l}\text { Classification of Tomato plant } \\
\text { disease }\end{array}$ & Improve computational time \\
\hline Oyewola et al. [36] & $\begin{array}{l}\text { deep residual neural net- } \\
\text { work }\end{array}$ & $\begin{array}{l}\text { Cassava Dis- } \\
\text { ease Dataset } \\
\text { from Kaggle }\end{array}$ & $\begin{array}{l}\text { cassava mosaic disease classifi- } \\
\text { cation }\end{array}$ & $\begin{array}{l}\text { Novel image augmentation methods } \\
\text { combined with other deep neural net- } \\
\text { works to improve accuracy }\end{array}$ \\
\hline Alli et al. [37] & MobileNetV2 & own data & Cassava disease recognition & $\begin{array}{l}\text { multi-class detection for identifying a va- } \\
\text { riety of plant diseases }\end{array}$ \\
\hline Almadhor et al. [35] & Bagged Tree classifier & own data & Guava fruit disease detection & $\begin{array}{l}\text { employing deep learning methods to ex- } \\
\text { tract features automatically }\end{array}$ \\
\hline Kundu et al. [38] & Custom-Net & own data & $\begin{array}{l}\text { pearl millet disease classifica- } \\
\text { tion }\end{array}$ & $\begin{array}{l}\text { scope of making the predictions based } \\
\text { on the parametric dataset collected by } \\
\text { the data collector part }\end{array}$ \\
\hline
\end{tabular}

In this work, the proposed CNN models are used for the classification of plant species for the PlantVillage (PV) and Flavia datasets. The performance of the developed models are compared with the AlexNet model with transfer learning. The proposed models have less depth as compared to AlexNet. The proposed models are compact in size and require less training time, maintaining good accuracy. The main contributions of this work are as follows:

1. Three highly accurate and compact models namely, N1, N2, and N3 are proposed for plant leaves classification. The proposed models show high classification accuracy, and they require less training time;

2. The performance of the models is validated by employing them to classify leaves from challenging PV and Flavia datasets. The models exhibit high classification accuracy;

3. To validate the versatility of the proposed models, they are also employed in tomato leaves disease classification using images captured from mobile phone. The disease classification accuracy shows that the proposed models are well suited for both plant leaves classification and disease classification.

\section{Material and Methods}

The work discusses the classification of a plant with a newly developed compact $\mathrm{CNN}$ model and AlexNet with transfer learning. The nine classes belonging to nine different species of plant images of the PlantVillage database are used for the classification. Additionally, the 32 classes of the Flavia dataset are classified. Figure 1 shows the workflow for plant classification and validation. The PV and Flavia datasets are augmented separately, and images are resized to a required size. The input image size for the proposed models 
is $256 \times 256 \times 3$, and the input image size for AlexNet is $227 \times 227 \times 3$. The dataset is further split into $80-20 \%$ of training dataset and testing dataset. The proposed models and AlexNet are trained with the training dataset for the classification of the plant species. The trained model is used for validation with the testing data for the prediction of the new data class.

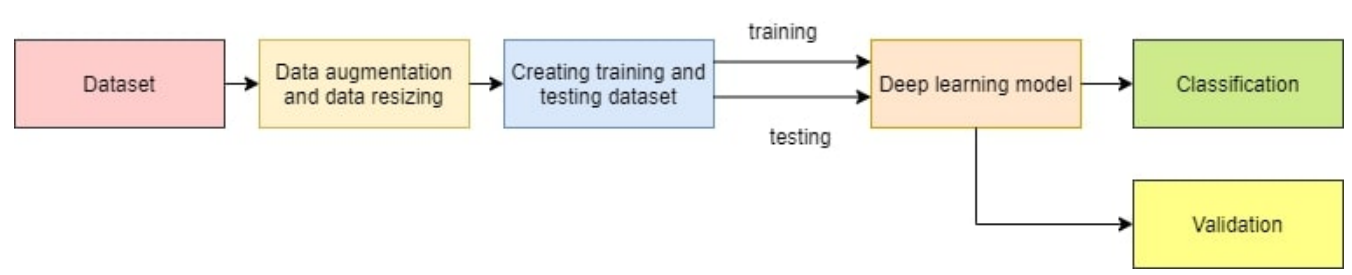

Figure 1. Proposed workflow for classification and validation of plant.

\subsection{Dataset of Plant Leaves}

A PV dataset includes images of healthy and diseased leaves of 38 different classes [23]. The dataset includes both healthy and diseased leaf categories of nine plant species, which include "apple, cherry, corn, grapes, peach, pepper, potato, strawberry, and a tomato" plant. We are considering the plant species here for classification purposes. The Flavia dataset [4] with 32 classes was used for classification with the proposed models. The Flavia dataset consists of a variant class of the plant leaf belonging to different crops like "Anhui Barberry", "Beale's barberry", "Big-fruited Holly", "Camphortree", "Canadian Poplar", "Castor Aralia", "Chinese Cinnamon", "Chinese Horse Chestnut”, "Chinese Redbud", "Chinese Toon", "Chinese Tulip Tree", "Crape Myrtle" or "Crepe Myrtle", "Deodar", "Ford Woodlotus", "Ginkgo Maidenhair Tree", "Glossy Privet", "Goldenrain Tree”, "Japan Arrowwood", "Japanese Cheesewood", "Japanese Flowering Cherry”, "Japanese Maple", "Nanmu", "Oleander", "Peach", "Pubescent Bamboo", "Southern Magnolia", "Sweet Osmanthus", "Tangerine”, “Trident Maple”, "True Indigo”, "Wintersweet”, "Yew Plum Pine".

\subsection{Dataset Pre-Processing}

Pre-processing the data is essential to maintain the uniformity and smooth functioning of the algorithm [24]. Deep learning behaves well when the input dataset is as large as possible and avoids overfitting. The very minute, invisible to human eye changes, such as adding noise and blur to the input images, can help CNNs learn more robust features $[39,40]$. In this work, the dataset is augmented with the Gaussian blur, salt and pepper noise with randomized scaling of 0.95 to 1.05 in a horizontal and vertical direction, and random rotation in the range $-30^{\circ}$ to $30^{\circ}$ of the images. The combination of augmentation used is shown in Table 3. The other augmentation performed here is with the rotation and flipping of the dataset. In position augmentation, images are rotated by $45^{\circ}, 135^{\circ}, 225^{\circ}$, and $315^{\circ}$ and flipped horizontally and vertically. The color augmentation of saturation, hue, and contrast is added. Saturation represents the amount of purity or colorfulness of a color. Hue represents the color (blue, red, green, etc.); its value ranges from 0 to 360. Histogram equalization is performed in evaluating the contrast value in color augmentation. Histogram equalization is known to improve accuracy.

The classification of plant leaves data is completed on a dataset with 38,400 images and an augmented dataset of 336,000 images. The deep learning network used in this work is proposed CNN model 1 (N1 model), CNN model 2 (N2 model), CNN model 3 (N3 model), and AlexNet model with transfer learning. The input images are resized to the size $256 \times 256 \times 3$ for the proposed developed models, and the images are resized to $227 \times 227 \times 3$ for the AlexNet model. 
Table 3. The amalgamation used to augment the dataset.

\begin{tabular}{|c|c|c|c|c|}
\hline \multicolumn{5}{|c|}{ Augmentation 1 (ad1) } \\
\hline Noise & Salt and pepper 1 & & & \\
\hline Blur & Gaussian blur & & & \\
\hline Position augmentation & Random scaling & random rotation & & \\
\hline \multicolumn{5}{|c|}{ Augmentation 2 (ad2) } \\
\hline Position augmentation & $\begin{array}{l}45^{\circ} \text { Rotation } \\
\text { Horizontal flip }\end{array}$ & $\begin{array}{l}135^{\circ} \text { Rotation } \\
\text { Vertical flip }\end{array}$ & $225^{\circ}$ Rotation & $315^{\circ}$ Rotation \\
\hline Color augmentation & Hue & Saturation & Contrast & \\
\hline
\end{tabular}

\subsection{Deep Learning Model}

The aim of this analysis is to create a computationally compact and accurate learning model for plant leaf classification. The proposed CNN model used in this work is developed with three convolutional layers as shown in Figure 2. The model consists of three sets of convolution 2D layer (Conv2D) followed by batch normalization layer and ReLU layer. There are three sets of Conv2D layer, batch normalization layer, and ReLU layer. The first two sets are followed by the max-pooling layer, and the third set is followed by the fully connected layer, softmax classifier, and classification layer. The convolution layer is modified with the size of the filter and the number of filters in the three CNN models of N1, N2, and N3, as shown in Table 4.

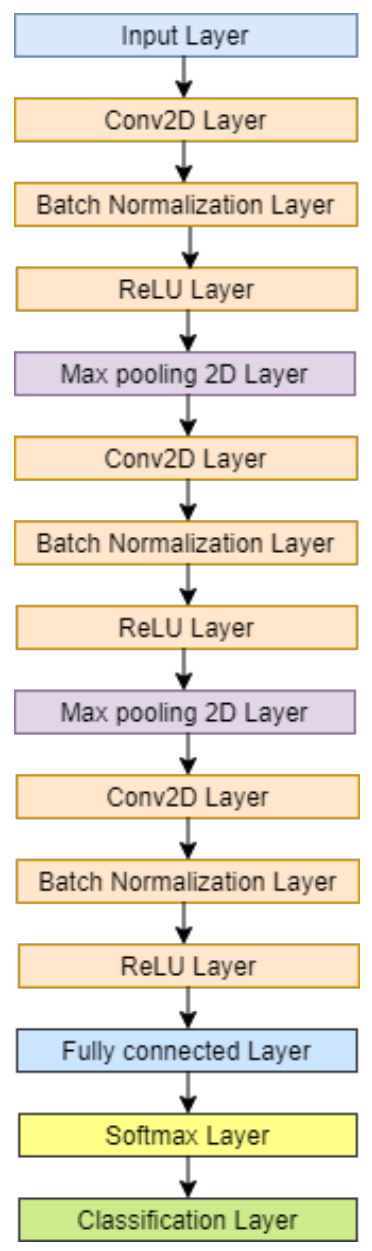

Figure 2. Proposed compact $\mathrm{CNN}$ model for classification and validation. 
Table 4. Convolution layers for N1 model, N2 model, and N3 model.

\begin{tabular}{cccc}
\hline CNN Layer & N1 Model & N2 Model & N3 Model \\
\hline 1st Conv2D & $3 \times 3,8$ & $3 \times 3,16$ & $7 \times 7,8$ \\
Maxpooling stride & 2 & 2 & 2 \\
2nd Conv2D & $3 \times 3,16$ & $3 \times 3,32$ & $5 \times 5,16$ \\
Maxpooling stride & 2 & 2 & 2 \\
3rd Conv2D & $3 \times 3,32$ & $3 \times 3,64$ & $3 \times 3,32$ \\
\hline
\end{tabular}

The convolutional layer specifies a set of filters that perform convolution across the entire image. Each convolutional layer in this architecture learns the various attributes that capture discriminatory patterns to differentiate the type of plant leaf. After each gradient update on a batch of data, Deep Neural Networks see different feature information from the previous layer. Furthermore, because the parameters of the previous layers are updated during the training phase, the data distribution of this input feature map varies greatly. This has a significant impact on training speed and necessitates the use of various heuristics to determine parameter initialization. The Rectified Linear Unit (ReLU) is an activation function commonly used in the design of neural networks, particularly CNNs. It is the identity function, $f(x)=x$, for all positive values of input ' $x$ ', and zeros out for negative values. ReLU is activated infrequently, mimicking the biological neuron's inactivity in response to certain impulses. This max-pooling layer only activates a subset of the neurons in the feature map. It is used across all blocks on a '2-by-2' window with a stride factor of ' 2 '. The feature maps' width and height are effectively reduced while the number of channels remains constant. In CNN models that predict a multinomial probability distribution, the softmax function is used as the activation function in the output layer. In other words, for multi-class classification problems, softmax is used as the classifier.

One of the benefits of small filter sizes over fully connected networks is that they minimize computing costs and weight sharing, resulting in lower back-propagation weights. Until now, the best choice for practitioners has been $3 \times 3[41,42]$. The $\mathrm{CNN}$ model N1 has a fixed filter size of $3 \times 3$ in all three convolution layers. In the 1st Conv2D, there are eight filters and, in the 2nd, Conv2D and 3rd Conv2D, there are 16 and 32 filters, respectively. In the CNN model N2, the filter size is kept the same as N1, but the number of filters in them is doubled as compared to N1. In the CNN model of N3, the filter size for the 1st Conv2D layer is $7 \times 7$, with eight filters. The 2 nd Conv2D layer is $5 \times 5$, with 16 filters, and the 3 rd Conv2D layer is $3 \times 3$ with 32 filters. The 1st Conv2D layer and 2nd Conv2D layer is followed by a max-pooling layer with a stride of 2 on a 2-by-2 window. The dataset is divided into training and testing datasets with the combination of $80-20 \%$ of the total data of 38,400 and 336,000 images. The data are trained with this combination for all the CNN models for the classification of the plant leaves. AlexNet is a pre-trained model that has the ability to classify up to 1000 classes [13]. In this work, we are classifying plant leaves of PV and Flavia dataset with 9 and 32 classes, respectively. For this purpose, AlexNet with transfer learning is used for classification. The objective of transfer learning is to optimise learning by leveraging the transferability of knowledge from the source [12]. All the models are implemented using the deep learning toolbox of MATLAB2019b in this study.

\subsection{Performance Parameters of the CNN Model}

The classification of the deep learning models is based on the performance and accuracy of the model. The confusion matrix of the test dataset is used for evaluating the performance parameters. The correct classification is shown by the diagonal elements and misclassification by non-diagonal elements of the confusion matrix. The elements of the confusion matrix are as follows [43]:

- "True Positive (TP): is the correctly labeled positive samples by the classifier";

- "True Negative (TN): is the correctly labeled negative samples by the classifier";

- "False Positive (FP): is the negative samples incorrectly labeled as positive"; and 
- "False Negative (FN): is the positive samples incorrectly labeled as negative".

The performance parameters evaluated here are macro recall, macro precision, macro F1 score, and mean accuracy [31]. Sensitivity/recall is the measure of the model that appropriately detects the positive class and is also known as the true positive rate. The model assigning positive events to the positive class is measured by a positive predictive value, also known as precision. F1 score is the harmonic mean of recall and precision. "Macro recall is the average per class effectiveness of a classifier to identify class labels". "Macro precision is an average per class agreement of the data class labels with those of the classifiers". "Macro F1 score is the relation between data's positive labels and those given by the classifier based on per class average". "Accuracy is the ratio of correct prediction by all predictions".

$$
\begin{aligned}
& \text { Sensitivity } / \text { Recall }=\frac{T P}{T P+F N} . \\
& \text { MacroRecall }=\frac{\sum_{n=1}^{C} \text { Sensitivity }}{C}
\end{aligned}
$$

where $C$ is the number of classes.

$$
\begin{gathered}
\text { Precision }=\frac{T P}{T P+F P} \\
\text { MacroPrecision }=\frac{\sum_{n=1}^{C} \text { Precision }}{C} \\
\text { F1score }=\frac{2 \times \text { Precision } \times \text { Recall }}{\text { Precision }+ \text { Recall }} \\
\text { MacroF1score }=\frac{\sum_{n=1}^{C} F 1 \text { score }}{C} \\
\text { Accuracy }=\frac{T P+T N}{T P+T N+F P+F N}
\end{gathered}
$$

There are nine classes in the PV dataset, so the confusion matrix metrics $9 \times 9$. In the case of the Flavia dataset with 32 classes, the confusion matrix metrics $32 \times 32$. For the N1 model, N2 model, N3 model, and AlexNet model, the accuracy of each class is evaluated. Each deep learning model's simulation time is noted. The time is in seconds, measured.

\subsection{Validation of the Trained CNN Model}

Validation of models is completed for the trained CNN models with the images from the PV and Flavia datasets, respectively, that was not part of the training or testing set. The validation of models is performed with 33,600 images. The validation of the model classifies the unknown image leaf data with its class and accuracy.

\section{Results and Discussion}

The PV dataset with nine species of plants is shown in Figure 3. The classes are abbreviated as follows. Apple plant with four varieties are A; Cherry with two varieties are Ch. Corn with four varieties are Co; Grape with four varieties are G. Peach with two varieties are Pch; Pepper with two varieties are Pep; potato with three varieties is Po. Strawberry with two varieties are $S$; tomato with nine varieties are To. 


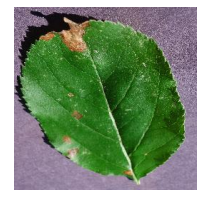

A

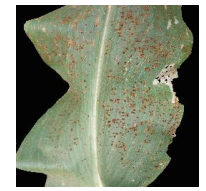

Co

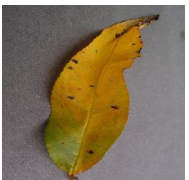

Pch

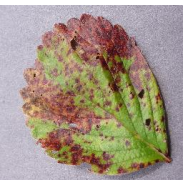

S

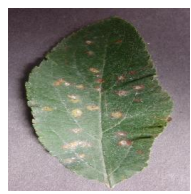

A

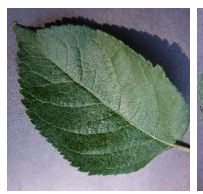

A

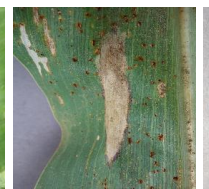

Co

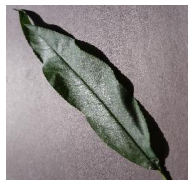

Pch

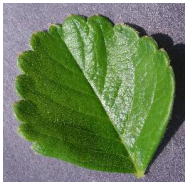

S

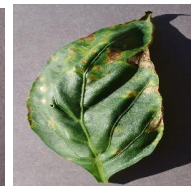

Pep

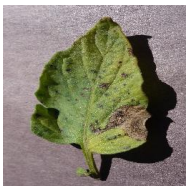

To

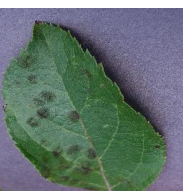

A

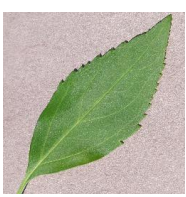

Ch

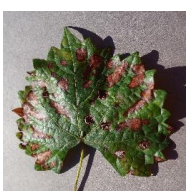

G

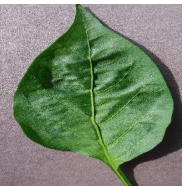

Pep

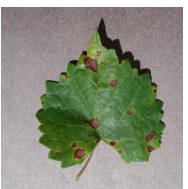

G

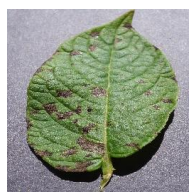

Po

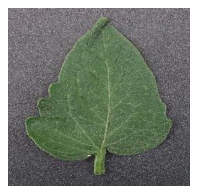

To

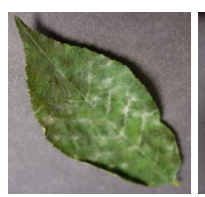

$\mathrm{Ch}$

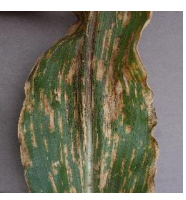

Co

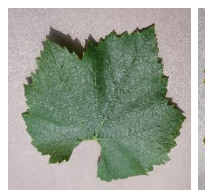

G

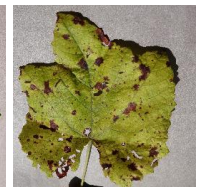

G

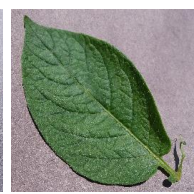

Po

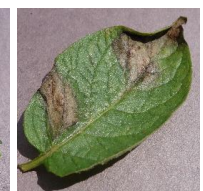

Po

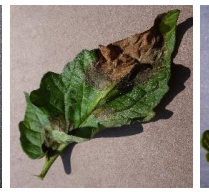

To

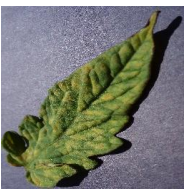

To

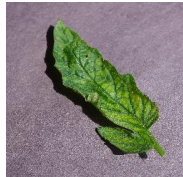

To

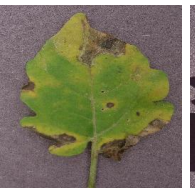

To

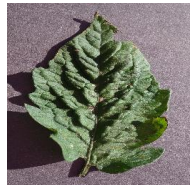

To

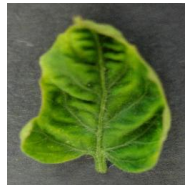

To

Figure 3. Plant leaf images from the training dataset of PV dataset.

The 32 classes of the Flavia dataset are shown in Figure 4. The classes are abbreviated as "Anhui Barberry" is AB, "Beale's Barberry" is BB, "Big-Fruited Holly" is BFH, "Castor Aralia" is CA, "Camphortree" is Cam, "Chinese Cinnamon" is CC, "Chinese Horse Chestnut" is CHC, "Crape Myrtle" is CM, "Canadian Poplar" is CP, "Chinese Redbud" is CR, "Chinese Toon" is CT, "Chinese Tulip Tree" is CTT, "Deodar" is D, "Ford Woodlotus" is FW, "Ginkgo Maidenhair Tree" is GMT, "Glossy Privet" is GP, "Goldenrain Tree" is GT, "Japan Arrowwood" is JA, "Japanese Cheesewood" is JC, "Japanese Flowering Cherry" is JFC, "Japanese Maple" is JM, "Nanmu" is N, "Oleander" is O, "Peach" is P, "Pubescent Bamboo" is PB, "Southern Magnolia" is SM, "Sweet Osmanthus" is SO, "Tangerine" is T, "Trident Maple" is TM, "True Indigo" is TI, "Wintersweet" is W, and "Yew Plum Pine" is YPP. All the 32 classes belong to different species here.

The pre-processing of the dataset is discussed in Section 2.2. The dataset is augmented with augmented data $1(\mathrm{ad} 1)$ and augmented data $2(\mathrm{ad} 2)$ and further resized to $256 \times 256 \times 3$ for the proposed models and $227 \times 227 \times 3$ for the AlexNet. Some of the data augmented images are shown in Figure 5. 


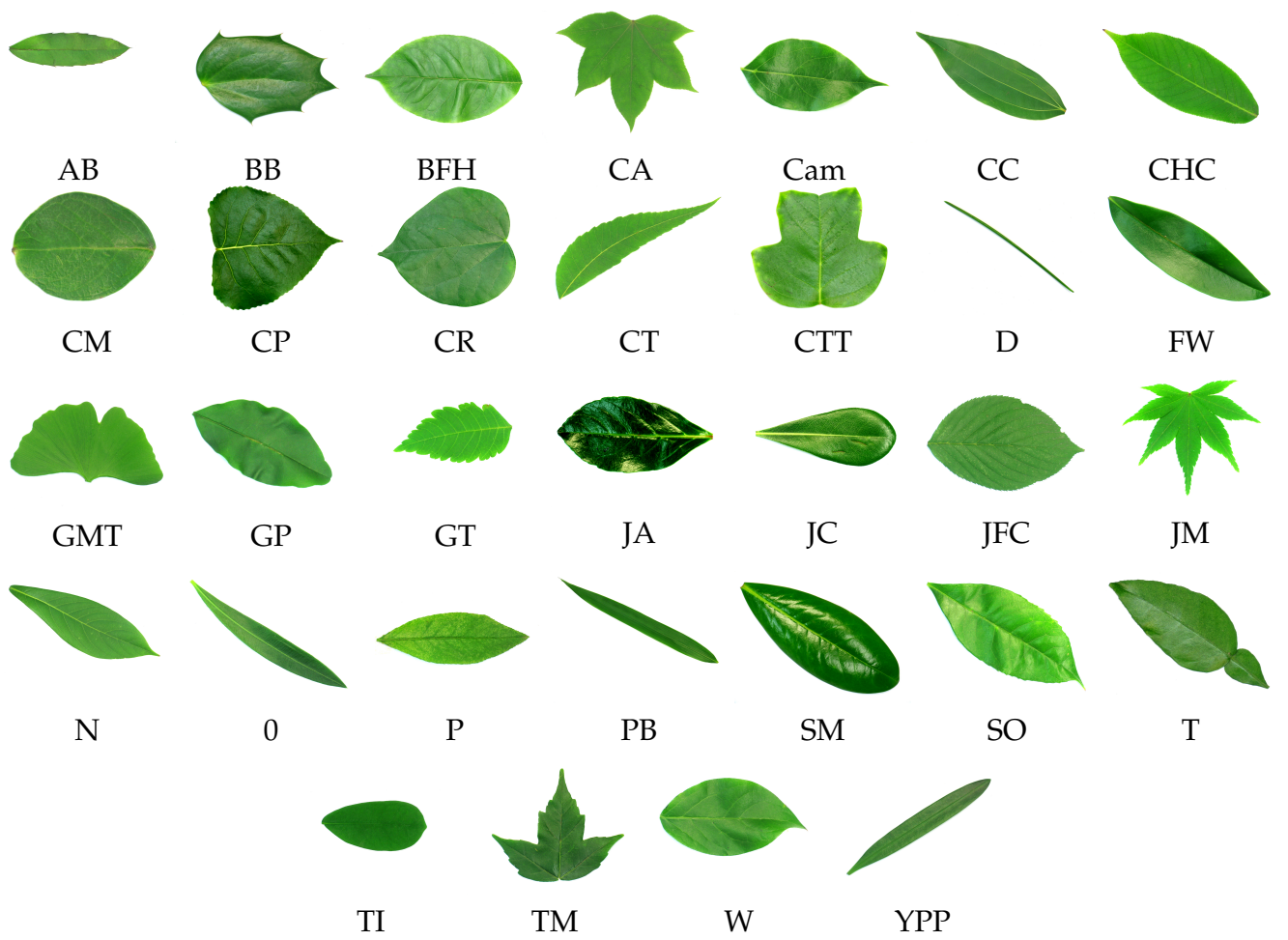

Figure 4. Plant leaf images from the training dataset of Flavia dataset.

Gaussian blur
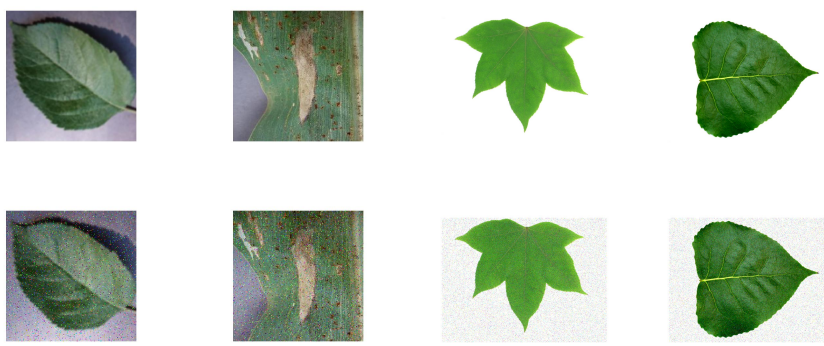

Random scaling and rotation
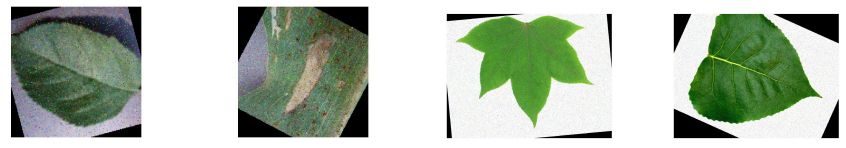

Contrast
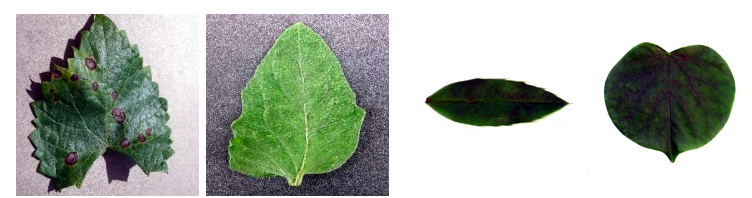

Hue
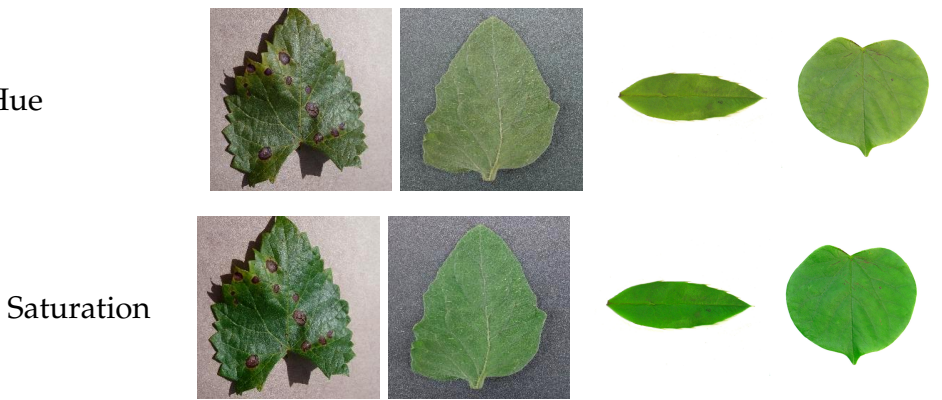

Figure 5. Some of the pre-processed images of PV and Flavia dataset. 
Classification of leaves of plants is performed using proposed compact models N1, $\mathrm{N} 2$, N3, and AlexNet with transfer learning. The classification accuracy of these models on a dataset, ad1, and ad2 is shown in Figure 6. The classification accuracy increases with the augmented dataset. More features are studied in the ad2, along with the increase in the number of images that are used for training the models. This helps in learning the model and achieving better prediction in terms of accuracy. The accuracy of the proposed N1 model is $86.58 \%$ with dataset and increased to $89.31 \%$ with ad 1 and $99.45 \%$ with ad2. The accuracy of the proposed N2 model is $92.09 \%$ with the dataset and increased to $99.65 \%$ with ad2. The accuracy of the proposed N3 model is $89.61 \%$ and increases to $89.8 \%$ with ad1 and $99.55 \%$ with ad2. AlexNet shows an accuracy of $98.53 \%$ with a dataset and increases to $99.73 \%$ with ad2. The accuracy of the N1 model, N2 model, N3 model, and AlexNet is almost the same for ad2. The time for training the model increases as the number of images increase. The training time for the N1 model, N2 model, N3 model, and AlexNet model is shown in Figure 7. The proposed N1, N2, and N3 models take less training time as compared to the AlexNet model. The number of layers and the size of the filter used in the proposed CNN N1 model, N2 model, and N3 model is less than the traditional AlexNet model. There are three $\mathrm{CNN}$ layers in the proposed developed model, whereas there are five Convolutional layers in AlexNet. The filter size is also more in AlexNet as compared to proposed developed models. The number of CNN layers and the size of the filters used in our developed model is compact as compared to AlexNet. This reduces the complexity of the model and so the training time required for the model is less.

Overfitting occurs when your model fits well on the training data, but it does not generalize well on new, unseen data. Overfitting problem can be prevented by taking measures such as data augmentation, simplifying the models, using dropout, regularization, and early stopping $[39,44,45]$. In this work, we have used two epochs for training the model. The learning rate for the model is 0.0001 . The training accuracy and training loss, along with the validation accuracy and validation loss, is as shown in Figure 8. The model with increasing training accuracy and validation accuracy and also decreasing training loss and validation loss shows that overfitting is prevented. The training accuracy and training loss are shown in Figure 8, (a) N1 model, (c) N2 model, (e) N3 model, and (g) AlexNet model. The validation accuracy and validation loss are shown in Figure 8, (b) N1 model, (d) N2 model, (f) N3 model, and (h) AlexNet model.

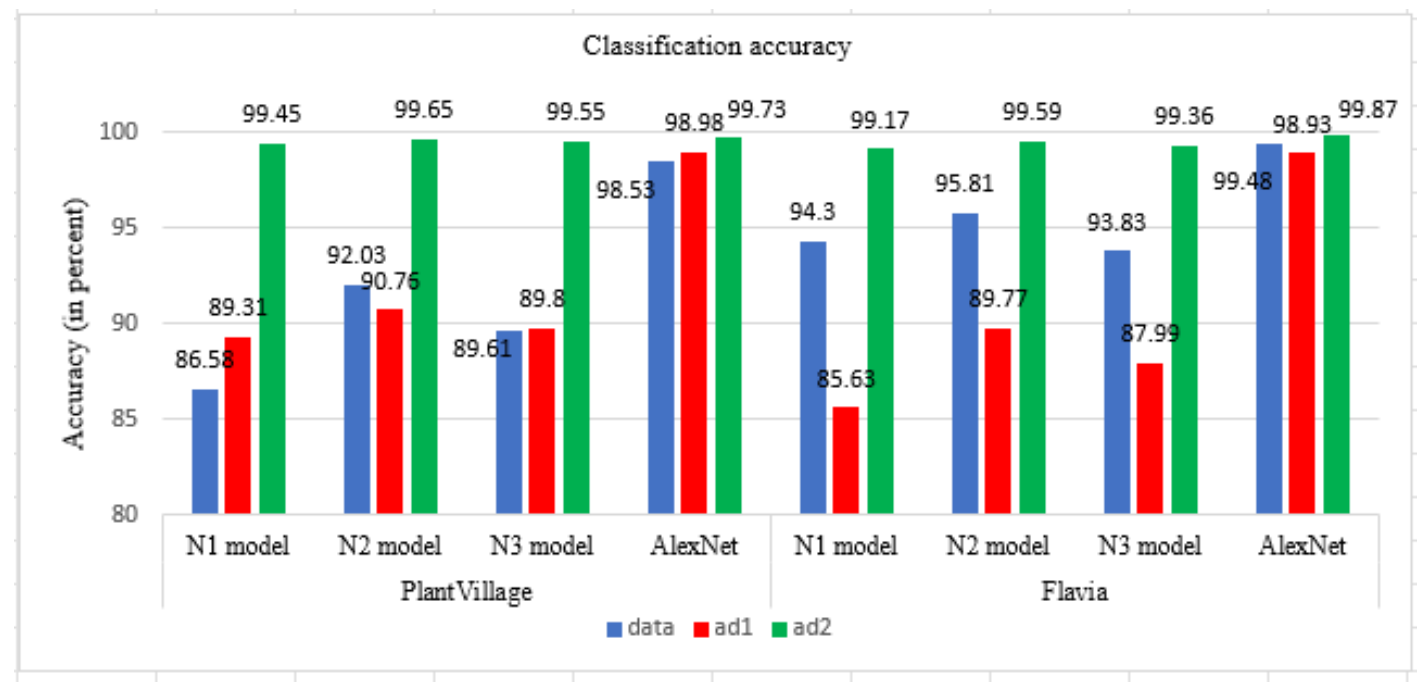

Figure 6. Classification accuracy of models for dataset, ad1 and ad2 for PV and Flavia dataset. 


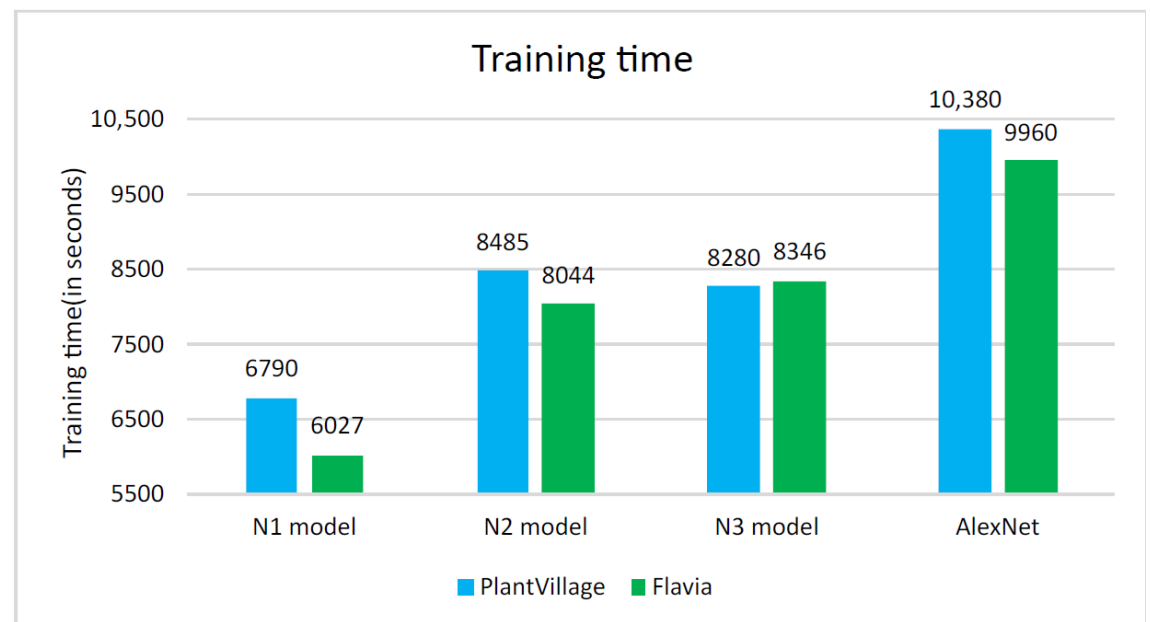

Figure 7. Training time of models for PV and Flavia dataset.

The comparison of models in terms of accuracy and size of the models with existing models are shown in Table 5. Jeon and Rhee [46] achieved an accuracy of $99.60 \%$ with GoogLeNet. Kaya et al. [47] in their work of plant classification, used PV and Flavia dataset with AlexNet and VGG16 models. Wang and Wang [48] classified plants with an accuracy of $84.47 \%$ with VGG16 and ResNet50 with $92.64 \%$. For the VGG16 and VGG 19 models, the accuracy achieved by of models is $81.3 \%$ and $96.25 \%$, respectively [ 49 ]. The combination of pruning and post-quantization was applied to VGG16, AlexNet, and LeNet model [50]. The pruning step was responsible for reducing the model size. The performance of models is $91.49 \%, 96.59 \%$, and $95.2 \%$, respectively. The ten-layer CNN model by [29] achieved an accuracy of $87.92 \%$ with the Flavia dataset and $84.02 \%$ with the PV dataset. The accuracy of the proposed N1 model is $99.45 \%$, proposed N2 model is $99.65 \%$, proposed N3 model is $99.55 \%$, and AlexNet with transfer learning is $99.73 \%$ with the models trained with ad2. The size of the proposed trained models is $14.8 \mathrm{MB}, 29.7 \mathrm{MB}$, and $14.8 \mathrm{MB}$, respectively, for the N1 model, N2 model, and N3 model as compared to AlexNet, which is $202 \mathrm{MB}$. The proposed N1 model and N3 model are $92.67 \%$ more compact than AlexNet, and the N2 model is $85.29 \%$ compact than AlexNet showing the same range accuracy results. The time for training the N1 model and N2 model is also less. The N1 model takes around 34.58\% less training time than AlexNet, and the N2 model takes around $18.25 \%$ less training time than AlexNet. N3 model takes $20.23 \%$ less training time than AlexNet.

The classified output images for the proposed N1 model, N2 model, N3 model, and AlexNet with transfer learning with $80 \%$ of training data for the PV dataset images are shown in Figure 9. The models are trained with a dataset, ad1, and ad 2 and their classified output is shown here. The classified output for the proposed models and AlexNet model is shown in Figure 9, (a) N1 model with the dataset, (b) N2 model with the dataset, (c) N3 model with dataset, (d) AlexNet model with the dataset, (e) N1 model with ad1, (f) N2 model with ad1, (g) N3 model with ad1, (h) AlexNet model with ad1, (i) N1 model with ad2, (j) N2 model with ad2, (k) N3 model with ad2, (l) AlexNet with ad2. The abbreviations used for the classified output images for the PV dataset are mentioned at the start of the Results and Discussion section. 


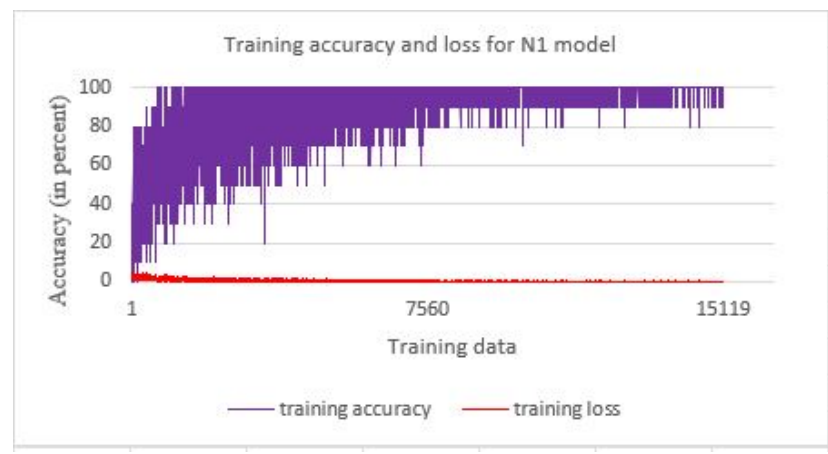

(a)

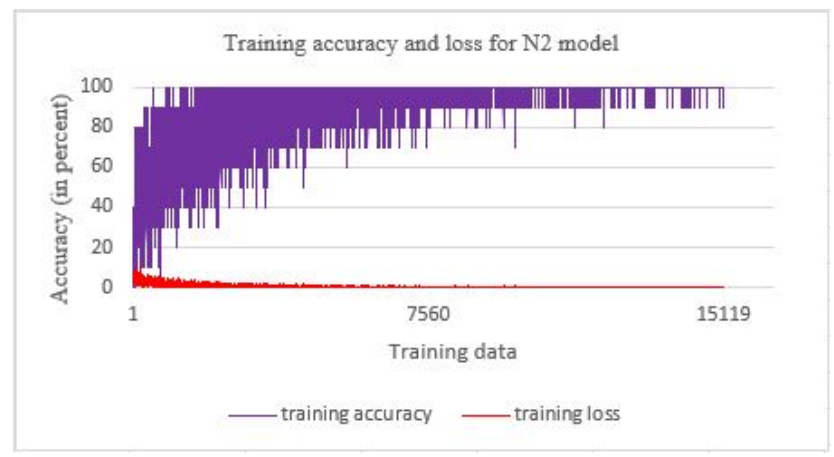

(c)

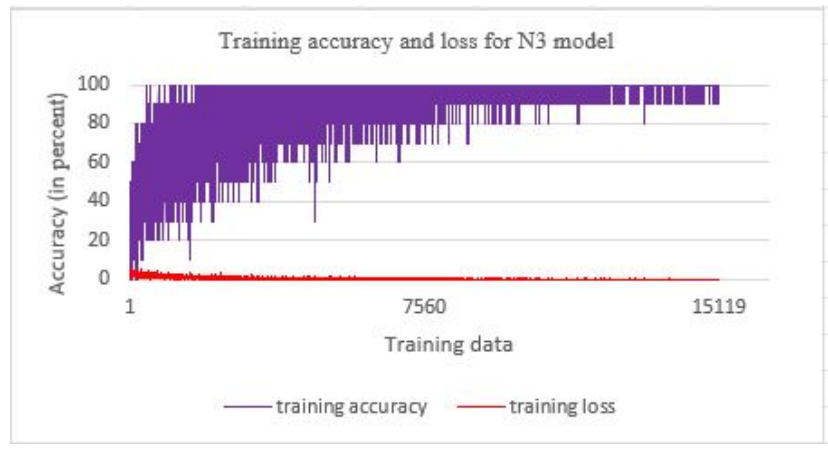

(e)

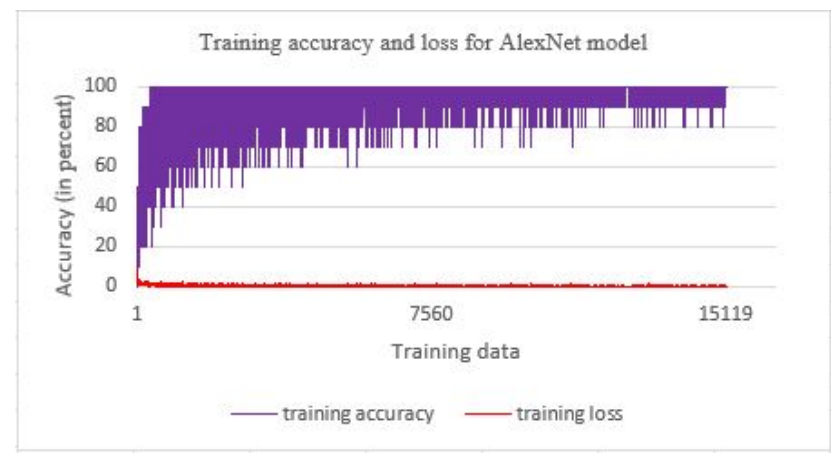

(g)

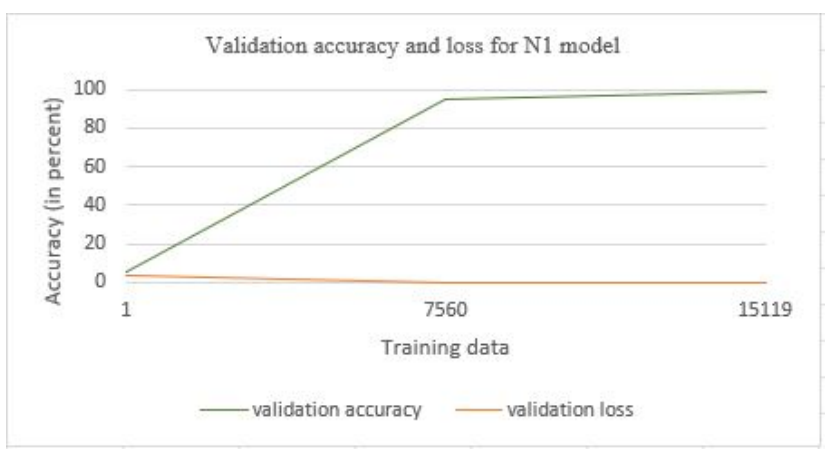

(b)

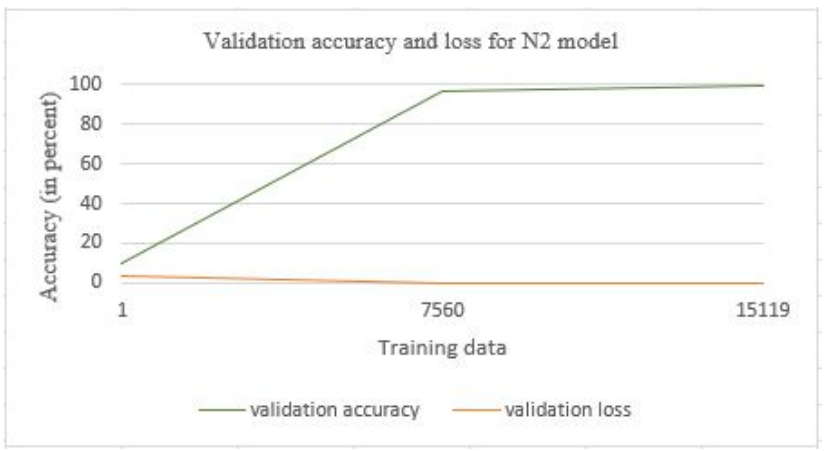

(d)

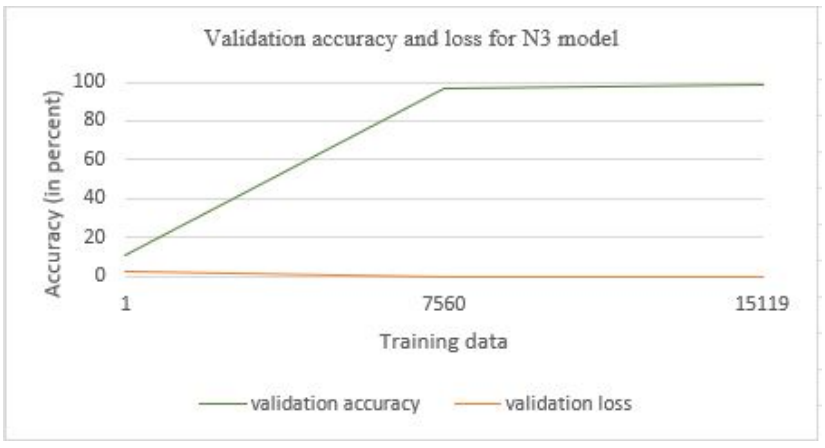

(f)

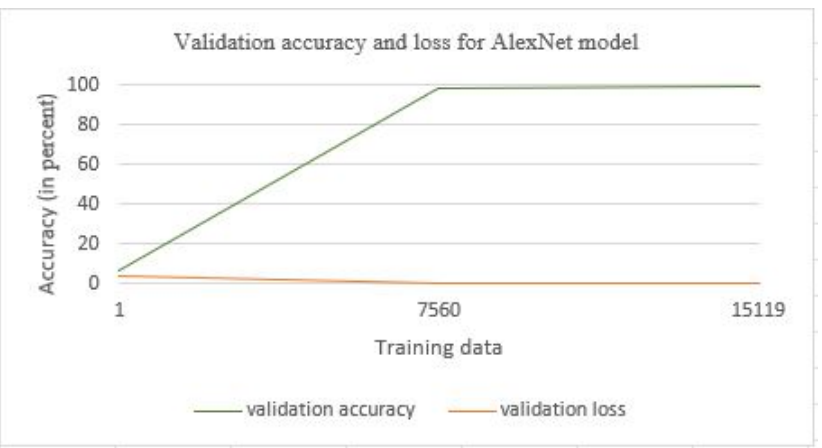

(h)

Figure 8. Training accuracy and training loss along with validation accuracy and validation loss for the N1 model, N2 model, N3 model, and AlexNet. 


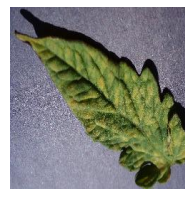

To

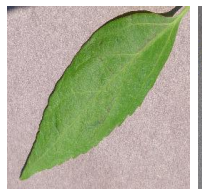

$\mathrm{Ch}$

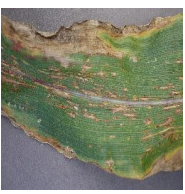

Co

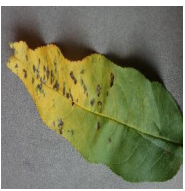

Pch

(a)

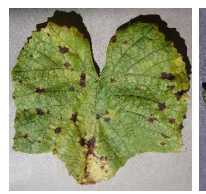

G

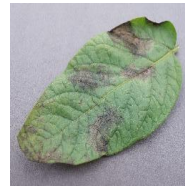

Po

(d)

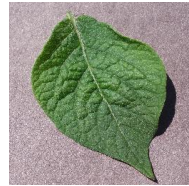

Po

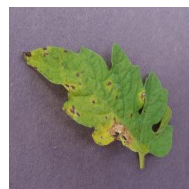

To

(g)

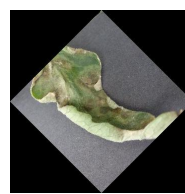

To

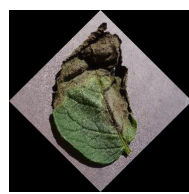

$\mathrm{Po}$

(j)

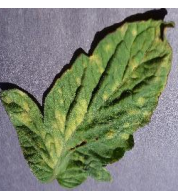

To

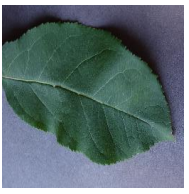

A

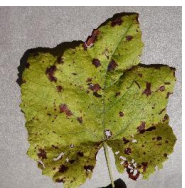

G

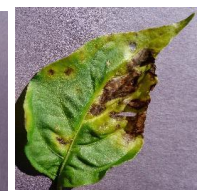

Pep

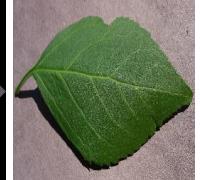

$\mathrm{Ch}$

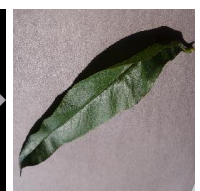

Pch

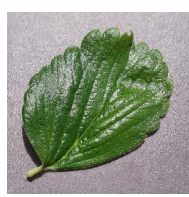

S

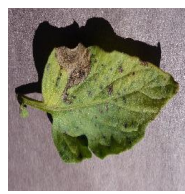

To

(b)

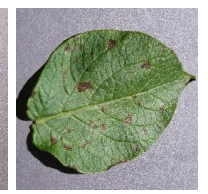

Po

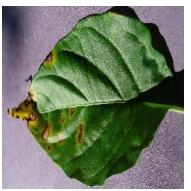

Po

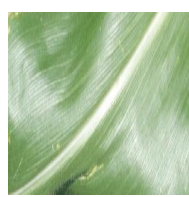

Co

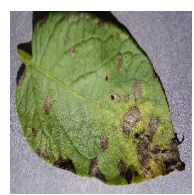

$\mathrm{Po}$

(e)

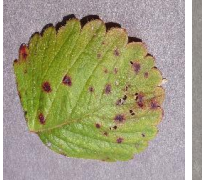

S

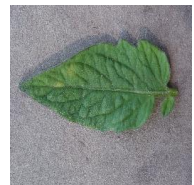

To

(h)

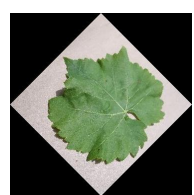

G

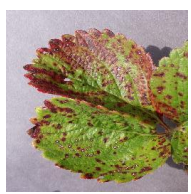

S

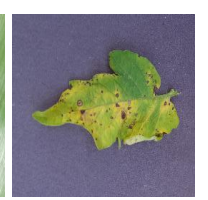

To

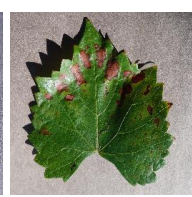

G

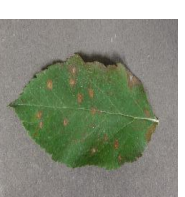

A

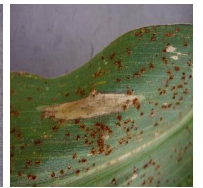

Co

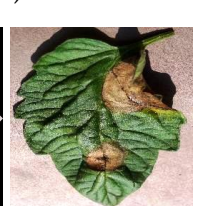

To

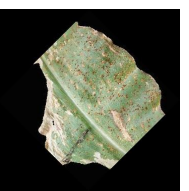

Co

(k)

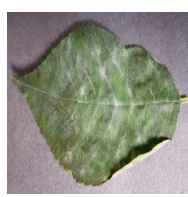

$\mathrm{Ch}$

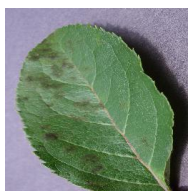

A

(c)

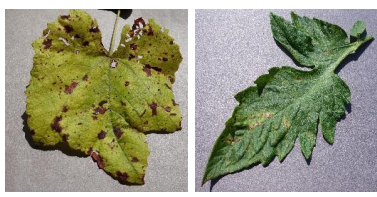

G

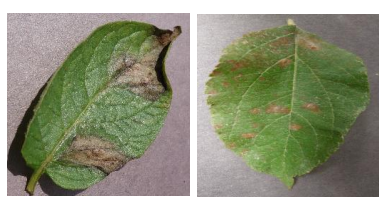

Po

(f)

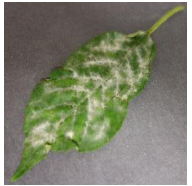

Ch

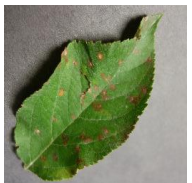

A

(i)

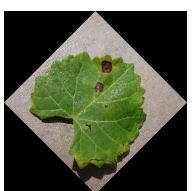

G

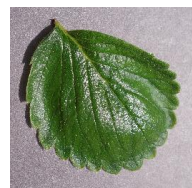

S

(1)

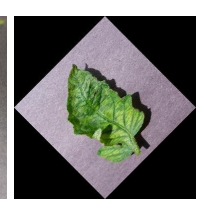

To

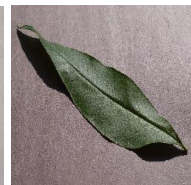

Pch

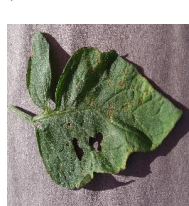

To

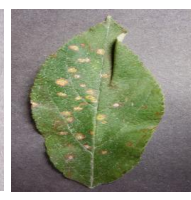

A

Figure 9. Classified output images for $80 \%$ training data with PV dataset using (a) N1 model with the dataset, (b) N2 model with the dataset, (c) N3 model with dataset, (d) AlexNet model with the dataset, (e) N1 model with ad1, (f) N2 model with ad1, (g) N3 model with ad1, (h) AlexNet model with ad1, (i) N1 model with ad2, (j) N2 model with ad2, (k) N3 model with ad2, (1) AlexNet with ad2. 
Table 5. Performance comparison of proposed work with other existing work in plant classification.

\begin{tabular}{|c|c|c|c|c|}
\hline Source & Dataset & Method & Accuracy & Size \\
\hline \multirow{2}{*}{ Mohanty et al. [23] } & \multirow{2}{*}{ PV } & AlexNet & $99 \%$ & $227 \mathrm{MB}[51]$ \\
\hline & & GoogLeNet & $99 \%$ & 27 MB [51] \\
\hline Lee et al. [10] & Flavia & AlexNet & $99.40 \%$ & $202 \mathrm{MB}$ [51] \\
\hline Jeon and Rhee [46] & PV & GoogLeNet & $99.60 \%$ & $27 \mathrm{MB}[51]$ \\
\hline \multirow{4}{*}{ Kaya et al. [47] } & \multirow{3}{*}{ Flavia } & Alexnet & $97.89 \%$ & 202 MB [51] \\
\hline & & VGG16 & $98.16 \%$ & $515 \mathrm{MB}$ [51] \\
\hline & & Alexnet & $98.6 \%$ & $202 \mathrm{MB}$ [51] \\
\hline & PV & VGG16 & $99.8 \%$ & $515 \mathrm{MB}[51]$ \\
\hline \multirow{2}{*}{ Wang [48] } & \multirow[b]{2}{*}{ Flavia } & VGG16 & $84.47 \%$ & $515 \mathrm{MB}[51]$ \\
\hline & & ResNet50 & $92.24 \%$ & $96 \mathrm{MB}[51]$ \\
\hline \multirow[b]{2}{*}{ Anubha Pearline et al. [49] } & \multirow[b]{2}{*}{ Flavia } & VGG16 & $95 \%$ & $515 \mathrm{MB}[51]$ \\
\hline & & VGG19 & $96.25 \%$ & $535 \mathrm{MB}$ [51] \\
\hline \multirow{2}{*}{ Venkatesh et al. [52] } & \multirow[b]{2}{*}{ PV } & VGG16 & $81.3 \%$ & $515 \mathrm{MB}$ [51] \\
\hline & & VGG16 +Inception & $92.2 \%$ & - \\
\hline \multirow{3}{*}{ Fountsop et al. [50] } & \multirow{3}{*}{ Flavia } & $\begin{array}{l}\text { VGG16 Pruning + } \\
\text { post-quantization }\end{array}$ & $91.49 \%$ & $36.76 \mathrm{MB}$ \\
\hline & & $\begin{array}{c}\text { AlexNet Pruning + } \\
\text { post-quantization }\end{array}$ & $96.59 \%$ & 32.37 MB \\
\hline & & $\begin{array}{l}\text { LeNet Pruning + } \\
\text { post-quantization }\end{array}$ & $95.02 \%$ & $9.91 \mathrm{MB}$ \\
\hline Liu et al. [29] & Flavia & ten-layer CNN & $87.92 \%$ & $7 \mathrm{MB}$ \\
\hline \multirow{8}{*}{ Proposed work } & \multirow{4}{*}{ PV } & N1 model & $99.45 \%$ & $14.8 \mathrm{MB}$ \\
\hline & & N2 model & $99.65 \%$ & $29.7 \mathrm{MB}$ \\
\hline & & N3 model & $99.55 \%$ & $14.8 \mathrm{MB}$ \\
\hline & & AlexNet & $99.73 \%$ & $202 \mathrm{MB}$ \\
\hline & \multirow{4}{*}{ Flavia } & N1 model & $99.17 \%$ & $14.8 \mathrm{MB}$ \\
\hline & & N1 model & $99.59 \%$ & $29.7 \mathrm{MB}$ \\
\hline & & N1 model & $99.36 \%$ & $14.8 \mathrm{MB}$ \\
\hline & & AlexNet & $99.87 \%$ & 202 MB \\
\hline
\end{tabular}

The classified output images for the proposed N1 model, N2 model, N3 model, and AlexNet with transfer learning with $80 \%$ of training data for the Flavia dataset images are shown in Figure 10. The models are trained with a dataset, ad1, and ad2 and their classified output is shown here. The classified output for the proposed models and AlexNet model is shown in Figure 10, (a) N1 model with the dataset, (b) N2 model with the dataset, (c) N3 model with dataset, (d) AlexNet model with the dataset, (e) N1 model with ad1, (f) N2 model with ad1, (g) N3 model with ad1, (h) AlexNet model with ad1, (i) N1 model with ad2, (j) N2 model with ad2, (k) N3 model with ad2, (l) AlexNet with ad2. The abbreviations used for the classified output images for the Flavia dataset are mentioned at the start of the Results and Discussion section.

The performance of the classification by the models trained with a dataset, ad1, and ad2, is evaluated on the PV dataset by confusion matrix as shown in Table 6 for the proposed N1 model, N2 model, N3 model, and AlexNet. The confusion matrix shows the information about classification and misclassification by the model. The diagonal elements show the correct classification, and the non-diagonal elements show the misclassification information. Table 6a shows the confusion matrix for the proposed N1 model trained for $80 \%$ dataset and tested for $20 \%$ of a dataset remaining. The diagonal elements show the correct classification of each class, and cells are colored in yellow. 

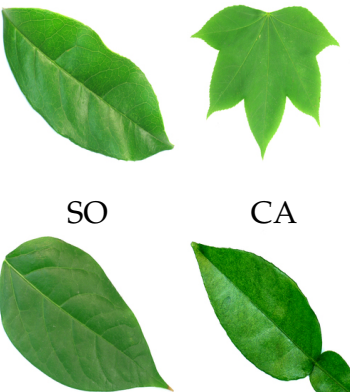

W

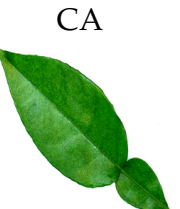

$\mathrm{T}$

(a)
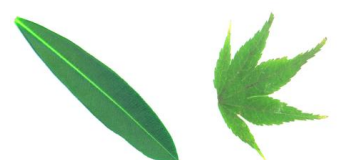

O
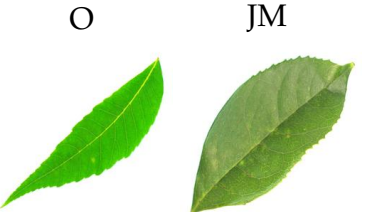

CT

(d)
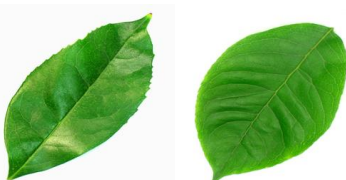

SO

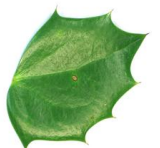

BB

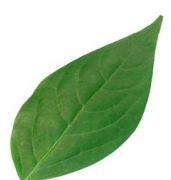

W

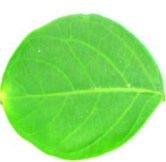

CM

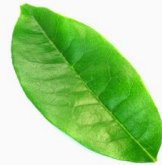

$\mathrm{SO}$

(b)
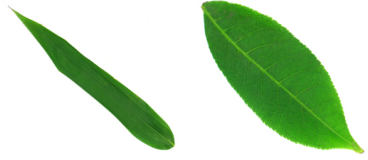

$\mathrm{CHC}$

(g)
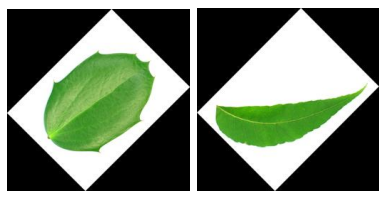

BB

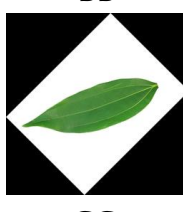

CC

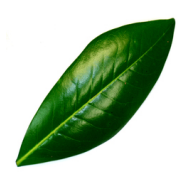

$\mathrm{SM}$

(e)

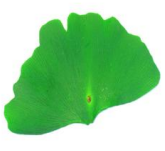

GMT

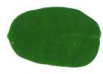

TI

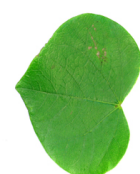

CR

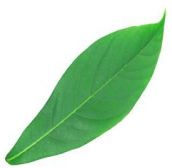

N

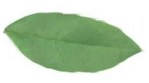

$\mathrm{AB}$

(h)

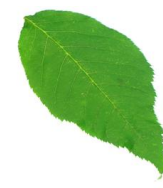

JFC

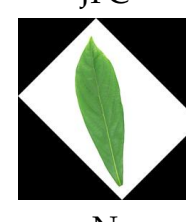

$\mathrm{N}$

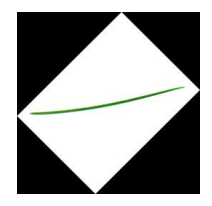

$\mathrm{D}$

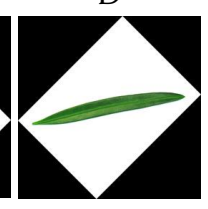

YPP

(k)

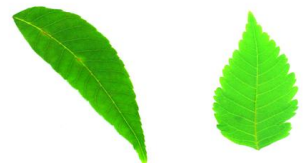

CT

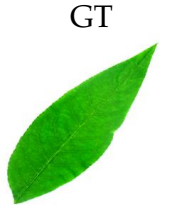

PB

(c)
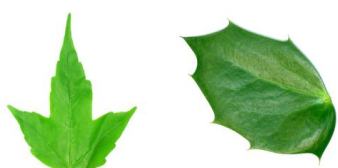

BB

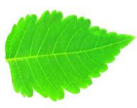

GT

(f)

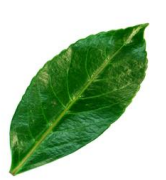

JA

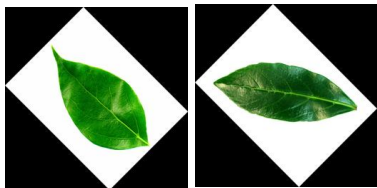

CAM

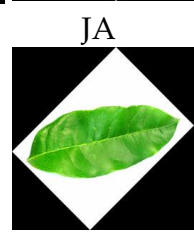

CTT

(j)
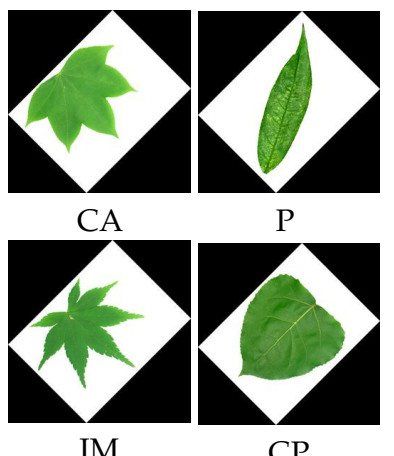

(1)

Figure 10. Classified output images for $80 \%$ training data with Flavia dataset using (a) N1 model with the dataset, (b) N2 model with the dataset, (c) N3 model with dataset, (d) AlexNet model with the dataset, (e) N1 model with ad1, (f) N2 model with ad1, (g) N3 model with ad1, (h) AlexNet model with ad1, (i) N1 model with ad2, (j) N2 model with ad2, (k) N3 model with ad2, (1) AlexNet with ad2. 
Table 6. Confusion matrix for proposed model for PV dataset.

(a) Confusion matrix for $\mathrm{N} 1$ using $80 \%$ of the training data with the dataset.

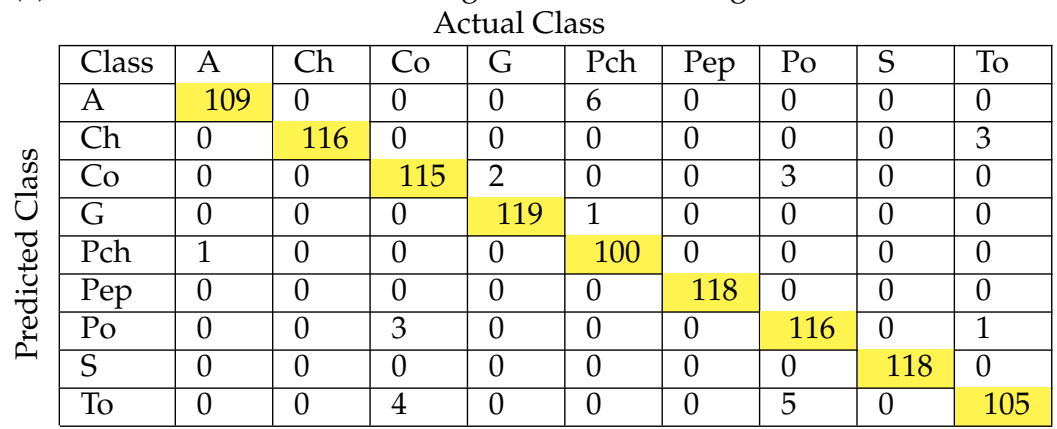

(b) The effect of data augmentation on confusion matrix of the model with ad1 and ad2 for PV dataset.

\begin{tabular}{|l|l|l|l|l|l|l|l|l|}
\hline \multirow{2}{*}{ Class } & \multicolumn{2}{|c|}{ N1 } & \multicolumn{2}{c|}{$\mathrm{N} 2$} & \multicolumn{2}{c|}{ N3 } & \multicolumn{2}{c|}{ AlexNet } \\
\cline { 2 - 9 } & ad1 & ad2 & ad1 & ad2 & ad1 & ad2 & ad1 & ad2 \\
\hline A & 4.2 & 15.1 & -10.4 & 3.7 & -5.8 & 7 & 0 & -0.1 \\
\hline Ch & 7.9 & 14.7 & -2.1 & 3 & -4.2 & 3.6 & 0.8 & 0.7 \\
\hline Co & 1.7 & 2.7 & 0.8 & 1 & 1 & 2 & 0 & 0 \\
\hline G & -1.2 & 2.8 & -5 & 1.2 & -0.4 & 2.8 & 0 & 0 \\
\hline Pch & 5.8 & 12.2 & 2.5 & 8.2 & 1 & 8.9 & 1.3 & 1 \\
\hline Pep & 10 & 24.3 & 11.3 & 21 & 3.8 & 18.1 & -0.8 & 0.4 \\
\hline Po & 5.4 & 11.1 & -2 & 3.5 & 8.3 & 11 & 0 & 0 \\
\hline S & 0 & 7 & 2.9 & 4 & -0.8 & 2 & 0 & -0.5 \\
\hline To & -4.6 & 4.7 & 2 & 6 & -0.4 & 7 & 0 & 0.7 \\
\hline
\end{tabular}

Correct classification

The effect of data augmentation on the confusion matrix of N1, N2, N3, and AlexNet models with ad 1 and ad2 is shown in Table $6 \mathrm{~b}$. The negative number shows a decrease in classification, and a positive sign indicates an increase in classification. The cell colored in green shows the increase in classification accuracy (percentage), and the cell colored in grey shows misclassification (percentage) after data augmentation.

It is seen that the performance of the model is improved with the models trained with an augmented dataset. The accuracy of the proposed N1 model for the PV dataset is improved by $7.9 \%$ and $14.7 \%$ for the "Ch" class for the model trained with ad 1 and ad2, respectively. The "Pep" class accuracy is improved by $10 \%$ and $24.3 \%$ for the model trained with ad1 and ad2, respectively. The performance of the proposed N2 model is improved by $11.3 \%$ and $21 \%$ for the "Pep" class for a model trained with ad 1 and ad2, respectively. The accuracy of the N2 model is improved by $2 \%$ and $6 \%$ with a model trained with ad 1 and ad2, respectively, for the "To" class. The accuracy of the proposed N3 model is improved by $8.3 \%$ and $11 \%$ for the "Po" class for the model trained with ad 1 and ad2, respectively. The "Pch" class accuracy is improved by $8.9 \%$ with ad2. The performance of the AlexNet model is improved by $1.3 \%$ for the "Pch" class for a model trained with ad1. In the case of AlexNet trained with ad2, the accuracy of two classes is reduced viz. "A" and "S." The accuracy performance for the proposed developed network N1 model, N2 model, and N3 model is seen to be improved for each of the classes with ad2.

The classification performance of the models trained with a dataset, ad1, and ad2 is evaluated on the Flavia dataset by confusion matrix as shown in Table 7 for the proposed N1 model. Table 7a shows the confusion matrix for the proposed N1 model trained for $80 \%$ dataset and tested for $20 \%$ of a dataset remaining. The diagonal elements show the correct classification of each class, and cells are colored in yellow. The effect of data augmentation on the confusion matrix of N1, N2, N3, and AlexNet models with ad1 and ad2 is shown in Table $7 \mathrm{~b}$. The negative number shows a decrease in classification, and a positive sign indicates an increase in classification. The cell colored in green shows the increase in 
classification accuracy (percentage), and the cell colored in grey shows misclassification (percentage) after data augmentation.

Table 7. Confusion matrix for proposed model for Flavia dataset.

(a) Confusion matrix for $\mathrm{N} 1$ using $80 \%$ of the training data with the dataset.

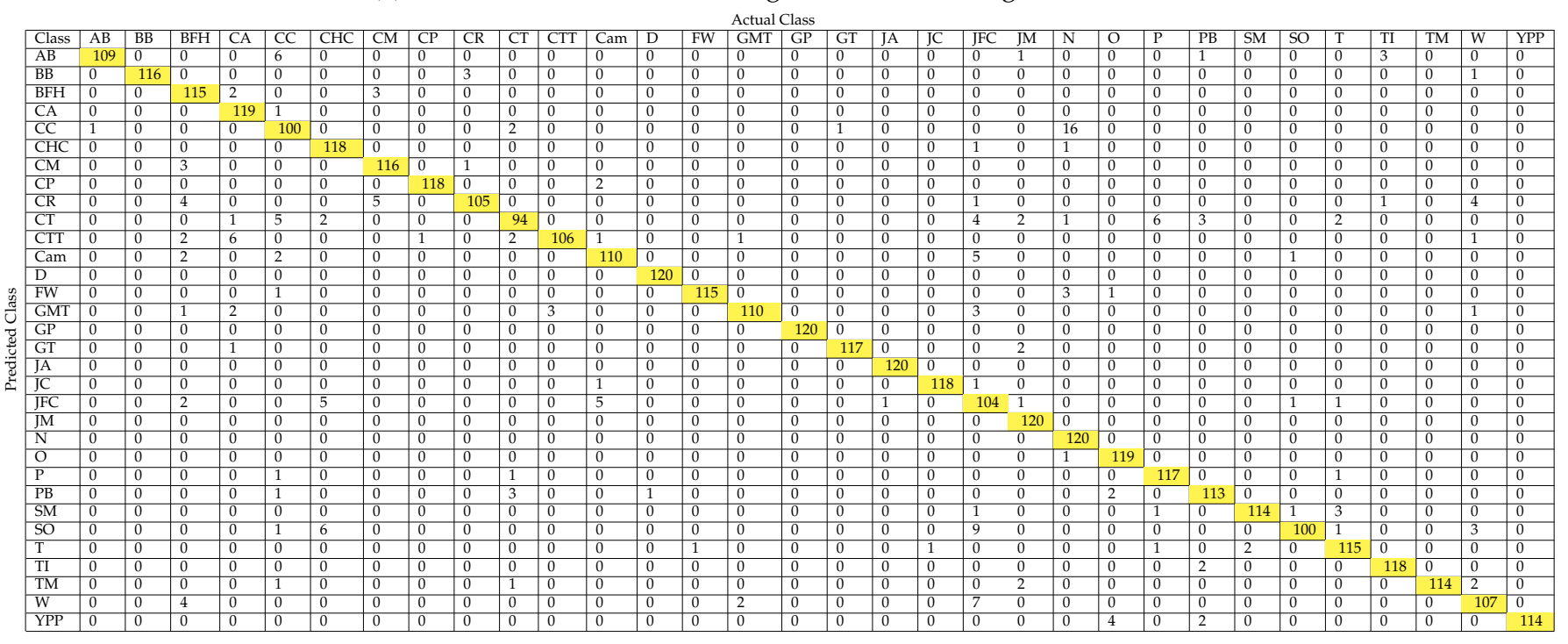

(b) The effect of data augmentation on confusion matrix of the model with ad1 and ad2 for PV dataset

\begin{tabular}{|l|l|l|l|l|l|l|l|l|}
\hline \multirow{2}{*}{ Class } & \multicolumn{2}{|c|}{$\mathrm{N} 1$} & \multicolumn{2}{c|}{$\mathrm{N} 2$} & \multicolumn{2}{c|}{$\mathrm{N} 3$} & \multicolumn{2}{c|}{ AlexNet } \\
\cline { 2 - 9 } & ad1 & ad2 & ad1 & ad2 & ad1 & ad2 & ad1 & ad2 \\
\hline AB & -5.8 & 8.9 & -15 & 5 & -2 & 5 & 0 & 0 \\
\hline BB & -5 & 2.3 & -2 & 1 & 2 & 3.3 & 0 & 0 \\
\hline BFH & 1.7 & 4.1 & 3.3 & 3 & 2 & 8 & 1 & 1 \\
\hline CA & -33.3 & 0.8 & -26.7 & 1.7 & -22 & 5 & 0 & 0 \\
\hline CC & -14.2 & 12.3 & -4.2 & 6.2 & -3 & 19.3 & 1 & -1 \\
\hline CHC & -7.5 & -0.2 & 3 & 4.6 & 0 & 2.6 & -0.8 & 2.3 \\
\hline CM & -0.8 & 3 & -2.5 & -0.2 & 3 & 4 & 2 & 2 \\
\hline CP & 0 & 1.4 & 0 & 2 & 0 & 2 & 0 & 0 \\
\hline CR & 5 & 12.4 & -5 & 1 & 10.8 & 16 & 0 & 0 \\
\hline CT & 7.5 & 20.4 & -10.8 & 5 & -8 & 9.9 & 0 & 0 \\
\hline CTT & 0.8 & 11.4 & -7.5 & 1.6 & -2.5 & 5 & 0 & 0 \\
\hline Cam & -10 & 8 & -10.8 & 2.4 & -9 & 10.4 & -3 & 0 \\
\hline D & -2.5 & 0 & -0.8 & 0 & -1 & 0 & 0 & 0 \\
\hline FV & 1.7 & -0.4 & 4.2 & 7.7 & 1.7 & -0.2 & -1 & 0 \\
\hline GMT & -2.5 & 8.1 & 7 & 13 & -11 & 9 & 0 & 0 \\
\hline GP & -0.8 & -1 & 0 & -0.5 & 0 & -1.2 & 0 & 0 \\
\hline GT & 0 & 1.9 & -0.8 & -1 & -1 & -1 & 0 & 0 \\
\hline JA & -24.2 & -0.6 & -12.5 & 0 & -18 & 1 & 0 & 0 \\
\hline JC & -17.5 & 0.5 & -12.5 & 3 & -28.3 & 3.5 & -1 & 0 \\
\hline JFC & -33.3 & 13 & -20.8 & 7.5 & -1.7 & 18.1 & 0 & 0 \\
\hline JM & -1.7 & -0.3 & 0.8 & 2.4 & 1 & 1 & 0 & 0 \\
\hline N & -11.7 & -0.1 & -12.5 & 3.8 & -13.3 & -1 & 0 & 0 \\
\hline O & -25 & 0.7 & -3.3 & 1.6 & -4 & 10.5 & -18 & 0 \\
\hline P & -0.8 & -1.1 & -4 & -2.5 & -0.8 & 1 & 1 & 1 \\
\hline PB & -26.7 & 5.2 & -22.5 & 0 & -17 & 11 & 2 & 1.7 \\
\hline SM & -20 & 4.2 & -12 & 6.3 & -9.2 & 4.6 & 0 & 0 \\
\hline SO & -2.5 & 16 & -20 & 3 & -18 & 3 & 0.8 & 0.7 \\
\hline T & -10 & 3.9 & -1.7 & 6 & -3 & 2.5 & 0 & 0 \\
\hline TI & 1.7 & 1.7 & 0 & 0 & 1 & 1 & 0 & 0 \\
\hline TM & 2.5 & 5 & 8 & 12 & -4 & 3 & -0.8 & 0 \\
\hline W & -27.5 & 10.8 & -16.7 & 11 & -30 & 16 & -6 & 0 \\
\hline YPP & -15 & 3.5 & 1.7 & 11.7 & -1.7 & 5.5 & 5.8 & 5.7 \\
\hline & & & & & & & & \\
\hline & & & & & 0.5 & 0 \\
\hline
\end{tabular}

Correct classification 
The accuracy of the proposed N1 model for the Flavia dataset is improved by $5 \%$ and $12.4 \%$ for the "CR" class for the model trained with ad1 and ad2, respectively, and 10.8\% and $16 \%$ for the proposed N2 model with ad1 and ad2. The "CT" class accuracy is improved by $7.5 \%$ with ad 1 and $20.4 \%$ with ad 2 for the proposed $\mathrm{N} 1$ model. The performance of the $\mathrm{N} 2$ model is improved by $7 \%$ and $13 \%$ for the "GMT" class for a model trained with ad1 and ad2, respectively. The accuracy of the proposed N3 model is improved by $19.3 \%$ for the "CC" class for the model trained with ad2. The performance of the AlexNet model is improved by $5.8 \%$ and $5.7 \%$ for the "YPP" class for a model trained with ad1 and ad2, respectively.

Data augmentation influences the average precision of the class [53,54]. Based on the confusion matrix, the performance parameters of macro recall, macro precision, macro F1 score, and mean accuracy are evaluated for the PV and Flavia datasets. The performance parameters of the proposed N1 model, N2 model, N3 model, and AlexNet is shown in Table 8 . The performance parameters of macro recall, macro precision, macro F1 score, and mean accuracy for the PV and Flavia dataset are compared here for data, ad1 and ad2. It is seen that the performance parameters are improved with the ad2. The proposed developed N1, N2, and N3 models have the same range performance as AlexNet. The size of these models is much more compact to AlexNet and gives great results.

Table 8. Performance parameters of N1 model, N2 model, N3 model, and AlexNet trained with data, ad1, and ad2.

\begin{tabular}{|c|c|c|c|c|c|c|}
\hline Dataset & Model & Data & $\begin{array}{l}\text { Macro } \\
\text { Recall }\end{array}$ & $\begin{array}{c}\text { Macro } \\
\text { Precision }\end{array}$ & $\begin{array}{c}\text { Macro } \\
\text { F1_score }\end{array}$ & $\begin{array}{c}\text { Mean } \\
\text { Accuracy }\end{array}$ \\
\hline \multirow{12}{*}{ PV } & \multirow{3}{*}{ N1 } & data & $87.76 \%$ & $86.58 \%$ & $86.57 \%$ & $99.16 \%$ \\
\hline & & ad1 & $89.50 \%$ & $89.31 \%$ & $89.26 \%$ & $99.33 \%$ \\
\hline & & $\mathrm{ad} 2$ & $99.45 \%$ & $99.45 \%$ & $99.45 \%$ & $99.97 \%$ \\
\hline & \multirow{4}{*}{ N2 } & data & $92.35 \%$ & $92.03 \%$ & $91.95 \%$ & $99.50 \%$ \\
\hline & & ad1 & $91.54 \%$ & $90.83 \%$ & $90.83 \%$ & $99.43 \%$ \\
\hline & & ad2 & $99.65 \%$ & $99.65 \%$ & $99.65 \%$ & $99.98 \%$ \\
\hline & & data & $90.20 \%$ & $89.61 \%$ & $89.57 \%$ & $99.35 \%$ \\
\hline & \multirow[t]{3}{*}{ N3 } & ad1 & $90.32 \%$ & $89.80 \%$ & $89.71 \%$ & $99.36 \%$ \\
\hline & & ad2 & $99.55 \%$ & $99.55 \%$ & $99.55 \%$ & $99.97 \%$ \\
\hline & & data & $98.60 \%$ & $98.53 \%$ & $98.52 \%$ & $99.91 \%$ \\
\hline & \multirow[t]{2}{*}{ AlexNet } & ad1 & $98.99 \%$ & $98.98 \%$ & $98.98 \%$ & $99.94 \%$ \\
\hline & & ad2 & $99.74 \%$ & $99.73 \%$ & $99.73 \%$ & $99.98 \%$ \\
\hline \multirow{12}{*}{ Flavia } & \multirow{4}{*}{ N1 } & data & $94.54 \%$ & $94.30 \%$ & $94.30 \%$ & $99.64 \%$ \\
\hline & & ad1 & $86.43 \%$ & $85.63 \%$ & $85.45 \%$ & $99.10 \%$ \\
\hline & & ad2 & $99.18 \%$ & $99.17 \%$ & $99.17 \%$ & $99.95 \%$ \\
\hline & & data & $96.05 \%$ & $95.91 \%$ & $95.91 \%$ & $99.74 \%$ \\
\hline & \multirow[t]{3}{*}{ N2 } & ad1 & $90.49 \%$ & $89.77 \%$ & $89.76 \%$ & $99.36 \%$ \\
\hline & & $\mathrm{ad} 2$ & $99.59 \%$ & $99.59 \%$ & $99.59 \%$ & $99.97 \%$ \\
\hline & & data & $93.99 \%$ & $93.83 \%$ & $93.80 \%$ & $99.61 \%$ \\
\hline & \multirow[t]{3}{*}{ N3 } & ad1 & $88.49 \%$ & $87.99 \%$ & $87.76 \%$ & $99.25 \%$ \\
\hline & & $\mathrm{ad} 2$ & $99.37 \%$ & $99.36 \%$ & $99.36 \%$ & $99.96 \%$ \\
\hline & & data & $99.50 \%$ & $99.48 \%$ & $99.48 \%$ & $99.97 \%$ \\
\hline & \multirow[t]{2}{*}{ AlexNet } & ad1 & $99.02 \%$ & $98.93 \%$ & $98.93 \%$ & $99.93 \%$ \\
\hline & & ad2 & $99.87 \%$ & $99.87 \%$ & $99.87 \%$ & $99.99 \%$ \\
\hline
\end{tabular}

For analyzing the results of the experimental designs by statistical tests, an analysis of variance (ANOVA) is developed by [55]. The ANOVA is performed on the performance parameters for the proposed models, and AlexNet trained with the dataset, ad1, and ad2 of both the datasets is shown in Tables 9-11. The parameters evaluated are Sum of Squares (SS), degree of freedom (df), mean squares (MS), $p$-value, F value, and F critical value. The condition for statistical significance is evaluated based on the $p$-value and if the $\mathrm{F}$ value is 
less than the F critical value. If the $p$-value is between 0.0001 to 0.001 , then it is extremely statistically significant when the $p$-value is between 0.001 to 0.01 , then it is very statistically significant when the $p$-value is between 0.01 to 0.05 , then it is statistically significant, and when the $p$-value is greater than 0.05 , then there is no statistical significance. In all three tables for ANOVA, we can see the statistical significance for the models evaluated on both datasets.

Table 9. ANOVA analysis of performance parameters evaluated for dataset.

\begin{tabular}{cccccccc}
\hline Source of Variation & SS & df & MS & F & $p$-Value & F Critical & Significance \\
\hline Dataset & 77.688 & 1 & 77.688 & 6.659 & 0.0164 & 4.259 & $* *$ \\
Models & 191.983 & 3 & 63.994 & 5.485 & 0.0051 & 3.009 & $* * *$ \\
Dataset $\times$ Models & 24.687 & 3 & 8.229 & 0.705 & 0.5582 & 3.009 & NS \\
Within & 279.985 & 24 & 11.666 & & & & \\
Total & 574.343 & 31 & & & & & \\
${ }^{* * *} p<0.001,{ }^{* *} p<0.01,{ }^{*} p<0.05 ; \mathrm{NS}, p \geq 0.05$. &
\end{tabular}

Table 10. ANOVA analysis of performance parameters evaluated for ad1.

\begin{tabular}{cccccccc}
\hline Source of Variation & SS & df & MS & F & $p$-Value & F Critical & Significance \\
\hline Dataset & 12.276 & 1 & 12.276 & 0.604 & 0.4446 & 4.259 & NS \\
Models & 366.819 & 3 & 122.273 & 6.015 & 0.0033 & 3.009 & *** \\
Dataset $\times$ Models & 7.659 & 3 & 2.553 & 0.125 & 0.944 & 3.009 & NS \\
Within & 487.848 & 24 & 20.327 & & & & \\
Total & 874.602 & 31 & & & & & \\
${ }^{* * *} p<0.001,{ }^{* *} p<0.01,{ }^{*} p<0.05 ; \mathrm{NS}, p \geq 0.05$. & & &
\end{tabular}

Table 11. ANOVA analysis of performance parameters evaluated for ad2.

\begin{tabular}{cccccccc}
\hline Source of Variation & SS & df & MS & F & $p$-Value & F Critical & Significance \\
\hline Dataset & $4.43 \times 10^{-6}$ & 1 & $4.43 \times 10^{-6}$ & 0.816 & 0.3752 & 4.259 & NS \\
Models & $6.23 \times 10^{-5}$ & 3 & $2.08 \times 10^{-5}$ & 3.829 & 0.0225 & 3.009 & $*$ \\
Dataset $\times$ Models & $1.13 \times 10^{-5}$ & 3 & $3.77 \times 10^{-6}$ & 0.696 & 0.5634 & 3.009 & NS \\
Within & 0.0001 & 24 & $5.42 \times 10^{-6}$ & & & & \\
Total & 0.0002 & 31 & & & & & \\
${ }^{* * *} p<0.001,{ }^{* *} p<0.01,{ }^{*} p<0.05 ; \mathrm{NS}, p \geq 0.05$. & & & & &
\end{tabular}

The ability of the trained model to classify new data is an important factor in decision making. The PV dataset has nine classes belonging to nine plant species. The classification of plant species for PV dataset images that were not part of the training and testing dataset is completed. The validation accuracy of the N1 model, N2 model, N3 model, and AlexNet models trained with PV dataset is shown in Table 12. The images that were not part of the training and testing dataset are used for validation of the model into respective species. The validation performance of pepper is lower compared to other species. The proposed N2 model classifies the apple species with 92.5\% accuracy, N2 model and AlexNet model classify cherry with 95\% accuracy. The validation accuracy of the N2 model and AlexNet is $97.5 \%$ for the corn. The validation of grape, peach, and strawberry is good for all the models. The validation accuracy of the N3 model for tomato is $91.11 \%$. Overall, the performance of the N2 model is more as compared to N1 and N3 models.

The classification of plant species for Flavia dataset images that were not part of the training and testing dataset is completed. In the case of the Flavia dataset, each of the 32 classes belong to different plant species. Validation accuracy of proposed N1 model, N2 model, N3 model, and AlexNet models trained with Flavia dataset is shown in Table 13. Almost all the species are showing good classification except for the Cam class. Overall, the N2 model is performing equally well as AlexNet. N2 model achieves better performance in classification, as well as validation for PV and Flavia dataset with compact model size. 
Table 12. Validation accuracy of proposed N1 model, N2 model, N3 model, and AlexNet models trained with PV dataset.

\begin{tabular}{ccccc}
\hline Species & N1 & N2 & N3 & AlexNet \\
\hline "Apple" & $82.5 \%$ & $92.5 \%$ & $90 \%$ & $100 \%$ \\
"Cherry" & $75 \%$ & $95 \%$ & $85 \%$ & $95 \%$ \\
"Corn" & $95 \%$ & $97.5 \%$ & $90 \%$ & $97.5 \%$ \\
"Grape" & $90 \%$ & $97.5 \%$ & $95 \%$ & $100 \%$ \\
"Peach" & $80 \%$ & $85 \%$ & $90 \%$ & $100 \%$ \\
"Pepper" & $40 \%$ & $60 \%$ & $70 \%$ & $100 \%$ \\
"Potato" & $73.33 \%$ & $90 \%$ & $86.67 \%$ & $100 \%$ \\
"Strawberry" & $100 \%$ & $90 \%$ & $100 \%$ & $100 \%$ \\
"Tomato" & $88.89 \%$ & $84.44 \%$ & $91.11 \%$ & $95.56 \%$ \\
\hline
\end{tabular}

Table 13. Validation accuracy of N1 model, N2 model, N3 model, and AlexNet models trained with Flavia dataset.

\begin{tabular}{|c|c|c|c|c|}
\hline Species & N1 & N2 & N3 & AlexNet \\
\hline $\mathrm{AB}$ & $100 \%$ & $60 \%$ & $80 \%$ & $100 \%$ \\
\hline $\mathrm{BB}$ & $100 \%$ & $100 \%$ & $100 \%$ & $100 \%$ \\
\hline BFH & $100 \%$ & $100 \%$ & $100 \%$ & $100 \%$ \\
\hline CA & $100 \%$ & $100 \%$ & $100 \%$ & $100 \%$ \\
\hline Cam & $10 \%$ & $10 \%$ & $10 \%$ & $50 \%$ \\
\hline $\mathrm{CC}$ & $60 \%$ & $50 \%$ & $50 \%$ & $100 \%$ \\
\hline $\mathrm{CHC}$ & $80 \%$ & $90 \%$ & $90 \%$ & $100 \%$ \\
\hline $\mathrm{CM}$ & $100 \%$ & $100 \%$ & $100 \%$ & $100 \%$ \\
\hline $\mathrm{CP}$ & $100 \%$ & $100 \%$ & $100 \%$ & $100 \%$ \\
\hline CR & $90 \%$ & $80 \%$ & $90 \%$ & $100 \%$ \\
\hline $\mathrm{CT}$ & $30 \%$ & $30 \%$ & $20 \%$ & $80 \%$ \\
\hline CTT & $100 \%$ & $100 \%$ & $100 \%$ & $100 \%$ \\
\hline $\mathrm{D}$ & $90 \%$ & $100 \%$ & $90 \%$ & $100 \%$ \\
\hline FW & $90 \%$ & $90 \%$ & $90 \%$ & $100 \%$ \\
\hline GMT & $100 \%$ & $100 \%$ & $100 \%$ & $100 \%$ \\
\hline GP & $100 \%$ & $100 \%$ & $100 \%$ & $100 \%$ \\
\hline GT & $80 \%$ & $50 \%$ & $70 \%$ & $100 \%$ \\
\hline JA & $100 \%$ & $100 \%$ & $100 \%$ & $100 \%$ \\
\hline $\mathrm{JC}$ & $50 \%$ & $50 \%$ & $60 \%$ & $80 \%$ \\
\hline JFC & $50 \%$ & $60 \%$ & $60 \%$ & $100 \%$ \\
\hline $\mathrm{JM}$ & $100 \%$ & $100 \%$ & $100 \%$ & $100 \%$ \\
\hline $\mathrm{N}$ & $90 \%$ & $90 \%$ & $90 \%$ & $100 \%$ \\
\hline $\mathrm{O}$ & $100 \%$ & $100 \%$ & $100 \%$ & $100 \%$ \\
\hline $\mathrm{P}$ & $100 \%$ & $100 \%$ & $100 \%$ & $100 \%$ \\
\hline PB & $50 \%$ & $70 \%$ & $60 \%$ & $90 \%$ \\
\hline SM & $40 \%$ & $70 \%$ & $40 \%$ & $100 \%$ \\
\hline $\mathrm{SO}$ & $70 \%$ & $60 \%$ & $70 \%$ & $90 \%$ \\
\hline $\mathrm{T}$ & $70 \%$ & $90 \%$ & $90 \%$ & $100 \%$ \\
\hline $\mathrm{TI}$ & $90 \%$ & $100 \%$ & $90 \%$ & $100 \%$ \\
\hline $\mathrm{TM}$ & $90 \%$ & $80 \%$ & $90 \%$ & $100 \%$ \\
\hline$W$ & $90 \%$ & $90 \%$ & $90 \%$ & $100 \%$ \\
\hline YPP & $30 \%$ & $90 \%$ & $20 \%$ & $70 \%$ \\
\hline
\end{tabular}

\section{Performance of Proposed Models for the Classification of Tomato Plant Disease}

The proposed model performed well in the classification of plant leaves. The proposed models are further used for classifying the disease in tomato plant leaf. The image data were collected from a tomato farm from Lavale, Pune, India. These data were captured with a mobile phone camera with the specification Super Speed Dual Pixel 12MP AF sensor. 
The images were captured with background around the tomato plant leaves. In real-life applications, input images cannot be expected to be of high quality. The images captured for each of the four classes were 300, which were augmented to 126,000 images. The augmented images are used to train the proposed models and AlexNet. The data of tomato plants are tomato early blight, leaf miner, and yellow leaf curl virus (YLCV) disease classes, along with healthy leaves. Figure 11 shows the tomato plant disease and a healthy class of Lavale farm dataset.

The classification accuracy of the proposed models and AlexNet is shown in Figure 12. The proposed N1 model achieves an accuracy of $99.864 \%$, N2 achieves $99.59 \%$, N3 achieves $99.63 \%$, and AlexNet achieves an accuracy of $99.35 \%$. The proposed N1 model takes $22.82 \%$, N2 model takes $56.18 \%$, and N3 model takes $25.73 \%$ less training time than AlexNet model. The N1 and N3 model are $99.06 \%$ and N2 model is $98.11 \%$ compact than AlexNet model. The proposed model's performance shows that they are capable of classifying the tomato plant disease images with complex backgrounds with good accuracy, less training time and compact model size. The proposed model's size is compact and takes less training time than the state of art models. The proposed models can be deployed as a stand-alone mobile app that will benefit the farmer with its results.

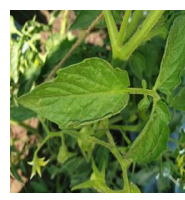

Healthy

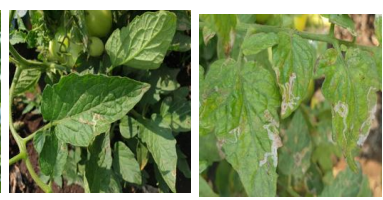

Early blight Leaf miner

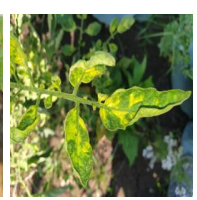

YLCV

Figure 11. Plant leaf images of Lavale data.

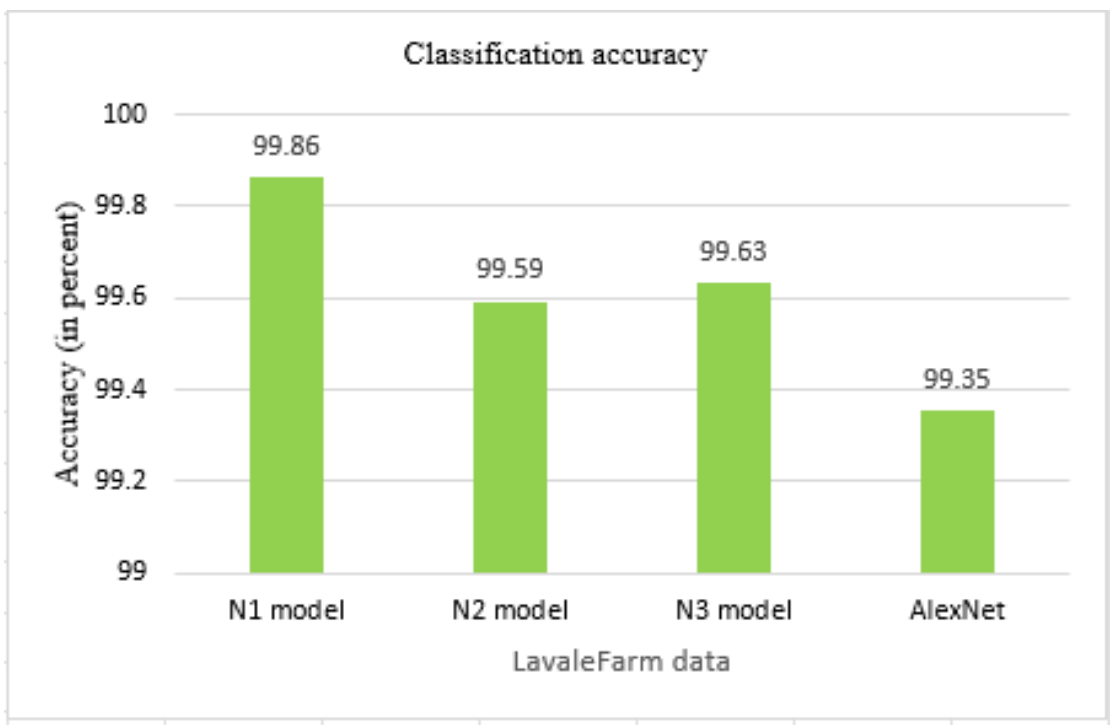

Figure 12. Classification accuracy of models for Lavale farm dataset.

\section{Conclusions}

The classification of Plant leaf images of nine classes of the PV database using proposed CNN models viz N1 model, N2 model, N3 model, and AlexNet with transfer learning is performed in this work. The developed model shows better performance after data augmentation is applied to them. The accuracy achieved by the proposed developed models is $99.45 \%$ with N1 model, $99.65 \%$ with N2 model, $99.55 \%$ with N3 model, and 99.73\% with the AlexNet model for the PV dataset. The accuracy achieved by the proposed developed models is $99.17 \%$ with N1 model, $99.59 \%$ with N2 model, $99.36 \%$ with N3 model, and $99.87 \%$ with the AlexNet model for the Flavia dataset with 32 classes. The accuracy of the developed models is equally good as AlexNet. The proposed N1 model and N3 model 
are $92.67 \%$ compacts than AlexNet, and N2 model is $85.3 \%$ compact than AlexNet. The training time of the developed model is reduced by $34.58 \%$ for the N1 model and $18.25 \%$ for the N2 model, and $20.23 \%$ for N3 as compared to the AlexNet. The N2 model has a compact size as compared to AlexNet and shows the same range accuracy. The classification of species is done by these trained models on the different images from PV and Flavia datasets that are not included in training and testing the models. The overall performance of the N2 model is more than N1 and N3 models. The experiments on two challenging datasets of PV and Flavia confirm the effectiveness of our method. The distinctiveness of proposed model classifies diseased plant leaves in the images captured with a mobile phone. The proposed models can be deployed as a stand-alone mobile app that will benefit the farmers as the proposed models are compact and give good classification results. The automatic plant classification will help in plant management thereby benefiting the society.

Author Contributions: Conceptualization, S.A.W. and R.H.; methodology, S.A.W.; software, S.A.W.; validation, R.H. and M.F.; formal analysis, S.A.W.; investigation, S.A.W.; resources, S.H.M.A.; data curation, S.A.W.; writing-original draft preparation, S.A.W. and R.H.; writing-review and editing, M.F. and S.H.M.A.; visualization, S.A.W.; supervision, R.H.; project administration, R.H.; funding acquisition, S.H.M.A. All authors have read and agreed to the published version of the manuscript.

Funding: This work is funded by Universiti Kebangsaan Malaysia under grant GUP-2020-009.

Institutional Review Board Statement: Not applicable.

Informed Consent Statement: Not applicable.

Data Availability Statement: Not applicable.

Conflicts of Interest: The authors declare no conflicts of interest.

\section{References}

1. Gao, Z.Y.; Xie, H.X.; Li, J.F.; Liu, S.L. Spatial-Structure Siamese Network for Plant Identification. Int. J. Pattern Recognit. Artif. Intell. 2018, 32, 1850035. [CrossRef]

2. Rehman, A.; Naz, S.; Razzak, M.I.; Akram, F.; Imran, M. A Deep Learning-Based Framework for Automatic Brain Tumors Classification Using Transfer Learning. Circuits Syst. Signal Process. 2019, 39, 757-775. [CrossRef]

3. Kaur, S.; Pandey, S.; Goel, S. Plants Disease Identification and Classification Through Leaf Images: A Survey. Arch. Comput. Methods Eng. 2019, 26, 507-530. [CrossRef]

4. Wu, S.G.; Bao, F.S.; Xu, E.Y.; Wang, Y.-X.; Chang, Y.-F.; Xiang, Q.-L. A Leaf Recognition Algorithm for Plant Classification Using Probabilistic Neural Network. In Proceedings of the 2007 IEEE International Symposium on Signal Processing and Information Technology, Cairo, Egypt, 15-18 December 2007; pp. 1-6.

5. Tiwari, S. A comparative study of deep learning models with handcraft features and non-handcraft features for automatic plant species identification. Int. J. Agric. Environ. Inf. Syst. 2020, 11, 44-57. [CrossRef]

6. Yang, K.; Zhong, W.; Li, F. Leaf segmentation and classification with a complicated background using deep learning. Agronomy 2020, 10, 1721. [CrossRef]

7. Priya, C.A.; Balasaravanan, T.; Thanamani, A.S. An efficient leaf recognition algorithm for plant classification using support vector machine. In Proceedings of the International Conference on Pattern Recognition, Informatics and Medical Engineering (PRIME-2012), Salem, India, 21-23 March 2012; pp. 428-432.

8. Du, J.X.; Zhai, C.M.; Wang, Q.P. Recognition of plant leaf image based on fractal dimension features. Neurocomputing 2013, 116, 150-156. [CrossRef]

9. Bodhwani, V.; Acharjya, D.P.; Bodhwani, U. Deep residual networks for plant identification. In Proceedings of the International Conference on Pervasive Computing Advances and Applications, Jaipur, India, 8-10 January 2019; pp. $186-194$.

10. Lee, S.H.; Chan, C.S.; Mayo, S.J.; Remagnino, P. How deep learning extracts and learns leaf features for plant classification. Pattern Recognit. 2017, 71, 1-13. [CrossRef]

11. Kumar, N.; Belhumeur, P.N.; Biswas, A.; Jacobs, D.W.; Kress, W.J.; Lopez, I.C.; Soares, J.V. Leafsnap: A Computer Vision System for Automatic Plant Species Identification. In Proceedings of the European Conference on Computer Vision, Firenze, Italy, 7-13 October 2012; pp. 502-516.

12. Duong-Trung, N.; Quach, L.D.; Nguyen, C.N. Learning deep transferability for several agricultural classification problems. Int. J. Adv. Comput. Sci. Appl. 2019, 10, 58-67. [CrossRef]

13. Krizhevsky, A.; Sutskever, I.; Hinton, G.E. ImageNet Classification with Deep Convolutional Neural Networks. In Proceedings of the Advances in Neural Information Processing Systems, Stateline, NV, USA, 3-8 December 2012; pp. 1-9. 
14. Villaruz, J.A. Deep convolutional neural network feature extraction for berry trees classification. J. Adv. Inf. Technol. 2021, 12, 226-233. [CrossRef]

15. Voulodimos, A.; Doulamis, N.; Doulamis, A.; Protopapadakis, E. Deep Learning for Computer Vision: A Brief Review. Comput. Intell. Neurosci. 2018, 2018, 7068349. [CrossRef] [PubMed]

16. Wang, G.; Sun, Y.; Wang, J. Automatic Image-Based Plant Disease Severity Estimation Using Deep Learning. Comput. Intell. Neurosci. 2017, 2017, 2917536. [CrossRef]

17. Yann, L.; Leon, B.; Yoshua, B.; Patrick, H. Gradient-Based Learning Applied to Document Recognition. Proc. IEEE 1998, 86, 2278-2324.

18. Szegedy, C.; Reed, S.; Sermanet, P.; Vanhoucke, V.; Rabinovich, A. Going deeper with convolutions. In Proceedings of the IEEE Conference on Computer Vision and Pattern Recognition, Providence, RI, USA, 16-21 June 2015; pp. 1-12.

19. He, K.; Zhang, X.; Ren, S.; Sun, J. Deep residual learning for image recognition. In Proceedings of the IEEE Conference on Computer Vision and Pattern Recognition, Las Vegas, NV, USA, 27-30 June 2016; pp. 770-778.

20. Simonyan, K.; Zisserman, A. Very deep convolutional networks for large-scale image recognition. In Proceedings of the 3rd International Conference on Learning Representations, San Diego, CA, USA, 7-9 May 2015; pp. 1-14.

21. Huang, G.; Liu, Z.; Maaten, L.V.D.; Weinberger, K.Q. Densely connected convolutional networks. In Proceedings of the IEEE Conference on Computer Vision and Pattern Recognition, College Park, MD, USA, 25-26 February 2017; pp. $2261-2269$.

22. Iandola, F.N.; Han, S.; Moskewicz, M.W.; Ashraf, K.; Dally, W.J.; Keutzer, K. SqueezeNet: AlexNet-level accuracy with 50x fewer parameters and $<0.5 \mathrm{MB}$ model size. In Proceedings of the 5th International Conference on Learning Representations, Toulon, France, 24-26 April 2017; pp. 1-13.

23. Mohanty, S.P.; Hughes, D.P.; Salathé, M. Using Deep Learning for Image-Based Plant Disease Detection. Front. Plant Sci. 2016, 7, 1419. [CrossRef] [PubMed]

24. Dyrmann, M.; Karstoft, H.; Midtiby, H.S. Plant species classification using deep convolutional neural network. Biosyst. Eng. 2016, 151, 72-80. [CrossRef]

25. Barré, P.; Stöver, B.C.; Müller, K.F.; Steinhage, V. LeafNet: A computer vision system for automatic plant species identification. Ecol. Inform. J. 2017, 40, 50-56. [CrossRef]

26. Haque, F.; Haque, S. Plant Recognition System Using Leaf Shape Features and Minimum Euclidean Distance. ICTACT J. Image Video Process. 2018, 9, 1919-1925. [CrossRef]

27. Zhu, Y.; Sun, W.; Cao, X.; Wang, C.; Wu, D.; Yang, Y.; Ye, N. TA-CNN: Two-way attention models in deep convolutional neural network for plant recognition. Neurocomputing 2019, 365, 191-200. [CrossRef]

28. Dileep, M.R.; Pournami, P.N. AyurLeaf: A Deep Learning Approach for Classification of Medicinal Plants. In Proceedings of the TENCON 2019-2019 IEEE Region 10 Conference (TENCON), Kochi, India, 17-20 October 2019; pp. 321-325.

29. Liu, J.; Yang, S.; Cheng, Y.; Song, Z. Plant Leaf Classification Based on Deep Learning. In Proceedings of the 2018 Chinese Automation Congress (CAC 2018), Xi'an, China, 30 November-2 December 2019; pp. 3165-3169.

30. Silva, P.F.B.; Marcal, A.R.S.; Silva, R.M.A.D. Evaluation of Features for Leaf Discrimination; Lecture Notes in Computer Science; Springer: Berlin/Heidelberg, Germany, 2013; pp. 197-204.

31. Brahimi, M.; Boukhalfa, K.; Moussaoui, A. Deep Learning for Tomato Diseases: Classification and Symptoms Visualization. Appl. Artif. Intell. 2017, 31, 299-315. [CrossRef]

32. Bharali, P.; Bhuyan, C.; Boruah, A. Plant Disease Detection by Leaf Image Classification Using Convolutional Neural Network. Inf. Commun. Comput. Technol. 2019, 1025, 194-205.

33. Ahmad, I.; Hamid, M.; Yousaf, S.; Shah, S.T.; Ahmad, M.O. Optimizing pretrained convolutional neural networks for tomato leaf disease detection. Complexity 2020, 2020, 8812019. [CrossRef]

34. Anandhakrishnan, T.; Jaisakthi, S.M. Identification of tomato leaf disease detection using pretrained deep convolutional neural network models. Scalable Comput. 2020, 21, 625-635.

35. Almadhor, A.; Rauf, H.T.; Lali, M.I.U.; Damaševičius, R.; Alouffi, B.; Alharbi, A. Ai-driven framework for recognition of guava plant diseases through machine learning from dslr camera sensor based high resolution imagery. Sensors 2021, 21, 3830. [CrossRef] [PubMed]

36. Oyewola, D.O.; Dada, E.G.; Misra, S.; Damaševičius, R. Detecting cassava mosaic disease using a deep residual convolutional neural network with distinct block processing. PeerJ Comput. Sci. 2021, 7, e352. [CrossRef]

37. Abayomi-Alli, O.O.; Damaševičius, R.; Misra, S.; Maskeliūnas, R. Cassava disease recognition from low-quality images using enhanced data augmentation model and deep learning. Expert Syst. 2021, 38, e12746. [CrossRef]

38. Kundu, N.; Rani, G.; Dhaka, V.S.; Gupta, K.; Nayak, S.C.; Verma, S.; Ijaz, M.F.; Woźniak, M. Iot and interpretable machine learning based framework for disease prediction in pearl millet. Sensors 2021, 21, 5386. [CrossRef] [PubMed]

39. Shorten, C.; Khoshgoftaar, T.M. A survey on Image Data Augmentation for Deep Learning. J. Big Data 2019, 6 , 60. [CrossRef]

40. Cygert, S.; Czyzewski, A. Toward robust pedestrian detection with data augmentation. IEEE Access 2020, 8, 136674-136683. [CrossRef]

41. Längkvist, M.; Jendeberg, J.; Thunberg, P.; Loutfi, A.; Lidén, M. Computer aided detection of ureteral stones in thin slice computed tomography volumes using Convolutional Neural Networks. Comput. Biol. Med. 2018, 97, 153-160. [CrossRef] [PubMed]

42. Jaiswal, S.; Nandi, G.C. Robust real-time emotion detection system using CNN architecture. Neural Comput. Appl. 2020, 32, 11253-11262. [CrossRef] 
43. Nweke, H.F.; Teh, Y.W.; Al-garadi, M.A.; Alo, U.R. Deep learning algorithms for human activity recognition using mobile and wearable sensor networks: State of the art and research challenges. Expert Syst. Appl. 2018, 105, 233-261. [CrossRef]

44. Moradi, R.; Berangi, R.; Minaei, B. A survey of regularization strategies for deep models. Artif. Intell. Rev. 2020, 53, 3947-3986 [CrossRef]

45. Srivastava, N.; Hinton, G.; Krizhevsky, A.; Sutskever, I.; Salakhutdinov, R. Dropout: A Simple Way to Prevent Neural Networks from Overfitting. J. Mach. Learn. Res. 2014, 15, 1929-1958.

46. Jeon, W.; Rhee, S. Plant Leaf Recognition Using a Convolution Neural Network. Int. J. Fuzzy Log. Intell. Syst. 2017, 17, 26-34. [CrossRef]

47. Kaya, A.; Keceli, A.S.; Catal, C.; Yalic, H.Y.; Temucin, H.; Tekinerdogan, B. Analysis of transfer learning for deep neural network based plant classification models. Comput. Electron. Agric. 2019, 158, 20-29. [CrossRef]

48. Wang, B.; Wang, D. Plant leaves classification: A few-shot learning method based on siamese network. IEEE Access 2019, 7, 151754-151763. [CrossRef]

49. Pearline, S.A.; Kumar, V.S.; Harini, S. A study on plant recognition using conventional image processing and deep learning approaches. J. Intell. Fuzzy Syst. 2019, 36, 1997-2004. [CrossRef]

50. Fountsop, A.N.; Fendji, J.L.E.K.; Atemkeng, M. Deep learning models compression for agricultural plants. Appl. Sci. 2020, 10, 6866. [CrossRef]

51. Deep Learning, MATLAB2019b. Available online: https://in.mathworks.com (accessed on 30 April 2021).

52. Venkatesh, Y.; Nagaraju, T.S.; Sahana, S.; Hegde, S.U. Transfer Learning based Convolutional Neural Network Model for Classification of Mango Leaves Infected by Anthracnose. In Proceedings of the 2020 IEEE International Conference for Innovation in Technology (INOCON), Begaluru, India, 6-8 November 2020; pp. 1-7.

53. Fuentes, A.; Yoon, S.; Kim, S.C.; Park, D.S. A Robust Deep-Learning-Based Detector for Real-Time Tomato Plant Diseases and Pests Recognition. Sensors 2017, 17, 2022. [CrossRef] [PubMed]

54. Fuentes, A.F.; Yoon, S.; Lee, J.; Park, D.S. High-Performance Deep Neural Network-Based Tomato Plant Diseases and Pests Diagnosis System With Refinement Filter Bank. Front. Plant Sci. 2018, 9, 1162. [CrossRef] [PubMed]

55. De Agustina, B.; Rubio, E.M.; Sebastián, M.Á. Surface roughness model based on force sensors for the prediction of the tool wear. Sensors 2014, 14, 6393-6408. [CrossRef] [PubMed] 